\title{
Down-Regulation of Circular RNA_000926 Attenuates Renal Cell Carcinoma Progression through miRNA-411-Dependent CDH2 Inhibition
}

\author{
Dong Zhang, Xiao-Jie Yang, Qi-Dong Luo, De-Lai Fu, Zhao-Lun Li, Peng Zhang, and Tie Chong
}

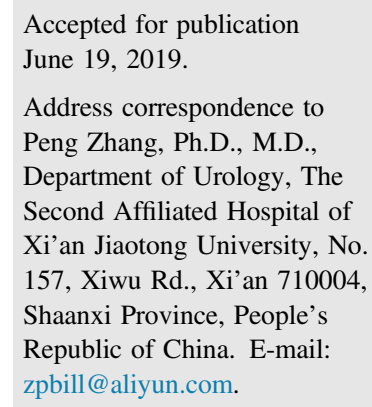

\begin{abstract}
Many studies have recognized that circular RNAs (circRNAs) can be promising targets for renal cell carcinoma (RCC) by acting as competing endogenous RNAs for miRNAs. This study intends to uncover the implication of a novel circRNA, circ_000926 in RCC, and how it affects tumorigenesis. Microarraybased circRNA/gene expression profiling of RCC was used to identify differentially expressed circRNAs/ genes in RCC and normal tissues. miRNAs targeting the screened circRNAs/genes were predicted online, followed by analyzing circ_000926 expression in RCC. The crosstalk among circ_000926, miRNA-411 (miR-411), and CDH2 was then validated. The expression of circ_000926, miR-411, and cadherin 2 (CDH2) was up-regulated or down-regulated in RCC cells to unearth their effects on the biological behaviors of RCC cells. circ_000926 was highly expressed in RCC tissues and cell lines, whereas CDH2 was verified to be a target of miR-411. As a competing endogenous RNA, circ_000926 could directly bind to miR-411 to up-regulate CDH2. Down-regulation of circ_000926 resulted in inhibited growth, migration, and invasion abilities of RCC cells, as well as suppressed epithelial-mesenchymal transition and tumor growth. However, the inhibition of miR-411 or elevation of $\mathrm{CDH} 2$ reversed the antitumor effects induced by silencing circ_000926. Down-regulation of circ_000926 exerts an inhibitory effect on RCC progression through miR-411-dependent $\mathrm{CDH} 2$ inhibition, highlighting a potential target for RCC treatment. (Am J Pathol 2019, 189: 2469-2486; https://doi.org/10.1016/j.ajpath.2019.06.016)
\end{abstract}

Approximately 270,000 individuals are diagnosed with kidney cancer every year, of whom 116,000 die of this disease. Ninety percentage of kidney cancer cases are due to renal cell carcinoma (RCC). ${ }^{1}$ RCC remains the most lethal among the common urologic cancers, and $20 \%$ to $30 \%$ of these patients have metastatic RCC. ${ }^{2}$ The pathologic characteristics of RCC involve a diverse range of histologic differences, therapeutic responses, and other genetic factors. ${ }^{3}$ Cigarette smoking behavior, obesity, hypertension, and/or related medications, together with germline mutations, are well-established risk factors for developing RCC. ${ }^{1,4}$ Although great advances have been made in the current systemic therapies for RCC, individualized and long-term therapy to help improve patient outcome remains a big challenge. ${ }^{5,6}$ Currently, the use of genomic and epigenomic data is an attractive method to help develop promising genetic and epigenetic diagnostic and prognostic indicators together with new targeted therapies for treating patients with $\mathrm{RCC}^{7}$

Circular RNAs (circRNAs) are stable RNA molecules that have specific developmental stage- and cell-specific expression patterns. $^{8}$ circRNAs usually function as miRNA sponges to modulate gene expression, ${ }^{9}$ which can be involved in different pathologic responses. circRNAs are useful biomarkers for cancer detection because of their potential diagnostic and prognostic values. ${ }^{10}$ miRNAs form an important component of the noncoding RNA family, which is

\footnotetext{
Supported by Free Exploration and Innovation Project of Xi'an Jiaotong University grant XJJ2018138 from the Exploration and Innovation Project of Xi' an Jiaotong University and Natural Science Basic Research Project of Shaanxi Province grant 2018JM7093.

D.Z. and X.-J.Y. contributed equally to this work.

Disclosures: None declared.
} 
involved in controlling multiple cell functions via suppressing the target genes. These genes are usually dysregulated in tumor microenvironment and cancer cells. ${ }^{11}$ One specific type of miRNA known as miRNA-411 (miR-411) has been clarified to be reduced and to act as a tumor suppressor in RCC. ${ }^{12}$ Moreover, miR-411 is also expressed poorly in breast cancer, and associated with histologic grade and lymph node metastasis. ${ }^{13}$ miR-411 was also identified as a tumor suppressor in colorectal cancer through posttranscriptional regulation of its target protein, PIK3R3. ${ }^{14}$ Cadherin $2(\mathrm{CDH} 2)$ is a classic cadherin and adhesion molecule that is mainly involved in tissue homeostasis, organogenesis, renal epithelial polarity, and integrity. ${ }^{15}$ It has been suggested that abundant expression of $\mathrm{CDH} 2$ is observed during the progression of epithelial-mesenchymal transition (EMT). ${ }^{16}$ Differentially expressed in different histologic subtypes of papillary $\mathrm{RCC}, \mathrm{CDH} 2$ represents a marker to differentiate papillary RCC type I and type II. ${ }^{17}$ Therefore, in this study, we predicted from the biological prediction websites that $\mathrm{CDH} 2$ was a target gene of miR-411. After screening out circRNA circ_000926 from the data set in the Gene Expression Omnibus (GEO) database (http://www. ncbi.nlm.nih.gov/geo; accession number GSE100186), $\mathrm{CDH} 2$ was highly expressed in clinical RCC tissues. This study focuses on investigating the regulatory effects of the circ_000926 on biological behaviors of RCC cells and its association with miR-411 and $\mathrm{CDH} 2$, with the aim of developing a feasible therapeutic target for RCC treatment.

\section{Materials and Methods}

\section{Ethics Statement}

The current study was performed under the approval of the Ethics Committee of the Second Affiliated Hospital of Xi' an Jiaotong University and strictly adhered to the Declaration of Helsinki. Informed consent was received from each patient participating in the study. All animal experiments and protocols were performed according to the guidelines of the Institutional Animal Ethics Committees.

\section{Microarray-Based circRNA Expression Profiling}

RCC-related circRNA/gene expression data sets were initially searched from The GEO database. Standardized pretreatment was performed using Affy package of R software version $1.63^{18}$ followed by differential analysis using Limma package of R software version 3.5 with $|\log 2 \mathrm{FC}|>$ 1.5 and adjusted $P<0.05$ as the threshold. ${ }^{19}$ A heat map of differentially expressed circRNAs/genes in RCC relative to matched normal tissues was then constructed.

\section{Study Participants and Sample Collection}

Eighty-five patients with RCC undergoing surgery at The Second Affiliated Hospital of Xi'an Jiaotong University from January 2014 to January 2017 were enrolled in the study. All patients received no drug therapy before sample collection. Collected RCC tissues and adjacent normal tissues were frozen in liquid nitrogen and stored for later use in subsequent experiments. ${ }^{20}$

RCC cell lines 786-O (ZY-H101), A498 (ZY-H401), 769-P (ZY-H100), and ACHN (ZY-H102) and normal kidney cell line HK-2 (ZY-H507) were obtained from Shanghai Zeye Biological Technology Co., Ltd. (Shanghai, China). The cell incubation was conducted by adding RPMI 1640 medium supplemented with $10 \%$ fetal bovine serum (FBS), $100 \mu \mathrm{g} / \mathrm{mL}$ of streptomycin, and $100 \mathrm{IU} / \mathrm{mL}$ of penicillin. Cells were subcultured at a ratio of 1:3 when cell confluence reached $80 \%$. Human RCC cell line screening for subsequent cell experiments was performed by determining hsa_circ_000926 expression using real-time quantitative RT-PCR (RT-qPCR).

\section{Cell Treatment}

A498 cells were collected and transfected with siRNA against circ_000926 (si-circ_000926; sequence: GTGGA AAAACTTTCTGCCTTA), miR-411 mimic, miR-411 inhibitor, $\mathrm{CDH} 2$ overexpression vector (oe-CDH2), or corresponding negative controls (NCs), including small interfering $\mathrm{NC}$ (si-NC), mimic $\mathrm{NC}$, inhibitor $\mathrm{NC}$, or overexpression NC (oe-NC), as well as their combined plasmids (si-NC plus inhibitor NC, si-circ_000926 plus inhibitor NC, $\mathrm{NC}$ mimic plus oe-NC, and miR-411 mimic plus oe-NC). All plasmids were purchased from Dharmacon Inc. (Lafayette, $\mathrm{CO}$ ). Collected cell suspension samples were seeded into sixwell plates at a density of $5 \times 10^{5}$ cells per well. Transfection was performed according to the specifications provided by the commercial Lipofectamine 2000 kit (Invitrogen, Carlsbad, CA). The medium was replaced 6 hours later. The cells were harvested 24 to 48 hours after transfection.

\section{RNA Extraction and Quantification}

After a 24-hour transfection period, a Trizol kit (Shanghai Haling Biological Technology Co., Ltd., Shanghai, China) was used for extraction of the total RNA from the RCC cells. The concentration, purity, and integrity of the RNA were determined by Nano-Drop ND-1000 spectrophotometry. The reverse transcription of total RNA into cDNA was performed based on the instructions provided by the EasyScript First-Strand cDNA Synthesis SuperMix kit (AE30102, TransGen Biotech Co., Ltd., Beijing, China). Specific cDNA of miR-411 was synthesized by TaqMan microRNA Reverse Transcription Kit (Thermo Fisher Scientific, Waltham, MA). RT-qPCR was conducted using SYBRPremix Ex TaqTM II kit (TaKaRa Biotechnology Co., Ltd., Dalian, China) in an ABI 7500 Real-Time PCR System (Applied Biosystems, Carlsbad, CA). The primers used are listed in Table 1 . The fold changes were calculated by means of relative quantification $\left(2^{-\Delta \Delta \mathrm{C}_{\mathrm{T}}}\right.$ method). 
Table 1 Primer Sequences for Real-Time Quantitative RT-PCR

\begin{tabular}{|c|c|}
\hline Item & Sequence \\
\hline \multirow[t]{2}{*}{ circ_000926 } & F: 5'-TTGTGCTTTCTGGAGGGTCT-3' \\
\hline & R: 5'-GCACAАATAАACCCСАСАТTTT-3' \\
\hline \multirow[t]{2}{*}{$\operatorname{miR}-411$} & F: 5'-GGTACTTGGAGAGATAGACCG-3' \\
\hline & R: 5'-GATACTGAGGGTTAGTGGACCG-3' \\
\hline \multirow[t]{2}{*}{ PCNA } & F: 5'-CGTAGCAGAGTGGTCGTTGTA-3' \\
\hline & R: 5'-TAGCTGGTTTCGGCTTCAGG-3' \\
\hline \multirow[t]{2}{*}{ MCM2 } & F: 5'-TCACGTGAACCACTTTTCGC-3' \\
\hline & R: 5'-AGGATTCCGATGATTCCGCC-3' \\
\hline \multirow[t]{2}{*}{ Ki67 } & F: 5'-GCCCCAACCAAAAGAAGTCT-3' \\
\hline & R: 5'-AGCTTTGTGCCTTCACTTCCA-3' \\
\hline \multirow[t]{2}{*}{$M M P-2$} & F: 5'-GACACATCTGGGCAGTTGCTA-3' \\
\hline & R: 5'-AACAGGTTGCAGCTCTCCTT-3' \\
\hline \multirow[t]{2}{*}{ MMP-9 } & F: 5'-CCTGGGCAGATTCCAAACCT-3' \\
\hline & R: $5^{\prime}$-GTACACGCGAGTGAAGGTGA-3' \\
\hline \multirow[t]{2}{*}{$N$-cadherin } & F: 5'-CGAATGGATGAAAGACCCATCC-3' \\
\hline & R: 5'-GGAGCCACTGCCTTCATAGTCAA-3' \\
\hline \multirow[t]{2}{*}{ E-cadherin } & F: 5'-GTACTTGTAATGACACATCTC-3' \\
\hline & R: 5'-TGCCAGTTTCTGCATCTTGC-3' \\
\hline \multirow[t]{2}{*}{ Vimentin } & F: 5'-GAAGAGAACTTTGCCGTTGAAG-3' \\
\hline & R: 5'-ACGAAGGTGACGAGCCATT- $3^{\prime}$ \\
\hline \multirow[t]{2}{*}{$\mathrm{CDH} 2$} & F: 5'-TGGGAAATGGAAACTTGATGGC-3' \\
\hline & R: 5'-AATCTGCAGGCTCACTGCTC-3' \\
\hline \multirow[t]{2}{*}{ U6 } & F: 5'-TCAGTTTGCTGTTCTGGGTG-3' \\
\hline & R: $5^{\prime}$-CGGTTGGCTGGAAAGGAG-3' \\
\hline \multirow[t]{2}{*}{ GAPDH } & F: 5'-CCTGGCCAAGGTCATCCATG-3' \\
\hline & R: 5'-GGAAGGCCATGCCAGTGAGC-3' \\
\hline
\end{tabular}

$F$, forward; $R$, reverse.

\section{Western Blot Analysis}

The cells were lysed using radioimmunoprecipitation lysis buffer. A bicinchoninic acid protein assay kit was used for extraction and quantification of total proteins. Proteins were then subjected to separation by $10 \%$ SDS-PAGE, followed by transfer onto nitrocellulose membranes. After blocking with $5 \%$ bovine serum albumin, the membrane was incubated with specific diluted primary rabbit polyclonal antibodies (Abcam Inc., Cambridge, MA): antibody CDH2 (ab76057, 1:1000), proliferating cell nuclear antigen (PCNA) (ab92552, 1:1000), minichromosome maintenance complex component 2 (MCM2) (ab4461, 1:1000), Ki-67 (ab92742, 1:1000), matrix metalloproteinase (MMP)-2 (ab92536, 1:1000), MMP-9 (ab76003, 1:1000), E-cadherin (ab40772, 1:10,000), and vimentin (ab92547, 1: 1000) overnight at $4^{\circ} \mathrm{C}$. On the next day, the membrane was incubated with horseradish peroxidase-conjugated goat anti-rabbit IgG (1:5000, Zhongshan Biotechnology Co., Ltd., Beijing, China). Finally, the immunocomplexes on the membrane were visualized using enhanced chemiluminescence (catalog number 808-25, Biomiga, San Diego, CA) and developed by exposure to radiographs (catalog number 36209ES01, Qcbio Science \& Technologies Co., Ltd., Shanghai, China). Glyceraldehyde-3phosphate dehydrogenase (GAPDH) was used as the internal control. The ratio of the gray value of the target band to GAPDH was representative of the relative protein expression.

\section{MTT Assay}

The cells were inoculated into 96-well plates at a density of $1 \times 10^{4}$ cells per well with six replicates after a transfection period of 48 hours. Next, $20 \mu \mathrm{L}$ MTT solution (Fortuneibotech Co., Ltd., Shanghai, China) was added into each well at the following time points and left to culture: 24th hour, 48th hour, and 72nd hour. After centrifugation, the supernatant was removed, and $100 \mu \mathrm{L}$ of dimethyl sulfoxide was added to each well, followed by a 10-minute oscillation on a micro-oscillator. After the purple crystal was completely dissolved, the OD values at $490 \mathrm{~nm}$ were evaluated using a microplate reader. The experiment was repeated three times independently.

\section{Clonogenic Assay}

The transfected cells were inoculated into six-well plates at a density of 400 cells per well and left to culture for 7 to 14 days. The culture medium was removed when colonies appeared visible. Cells were fixed with methanol and stained with $0.1 \%$ crystal violet. The number of newly formed colonies was counted and recorded.

\section{Scratch Test}

A498 cells were seeded into six-well plates at a concentration of $5 \times 10^{5}$ cells per well. Marker pens were used to draw lines evenly on the back of the plate. When cells covered the plate surface, the cells were starved for 12 hours with $1 \%$ FBS. A straight line on the cell surface was then drawn using a $200-\mu \mathrm{L}$ sterile pipette tip and ruler and rinsed three times with $2 \mathrm{~mL}$ of PBS to wash away the fallen cells. The plate was cultured with serum-free medium. Images were captured at 0 hour and 24 hours under an inverted microscope, and the scratch distance was measured.

\section{Transwell Assay}

After 48 hours of transfection, A498 cells were incubated in serum-free medium after 24 hours of starvation. The cells were resuspended with a density of $3 \times 10^{4}$ cells $/ \mathrm{mL}$. Subsequently, Transwell chambers coated with diluted Matrigel (1:8; Sigma-Aldrich, St. Louis, MO) were placed in 24-well plates. Cell suspension $(200 \mu \mathrm{L})$ was supplemented into the apical chamber of Transwell chamber coated with Matrigel, after which the chamber was cultured with $600 \mu \mathrm{L}$ of RPMI 1640 medium with $20 \%$ FBS. After 24 hours, the chamber was taken out, and the cells in the apical chamber were wiped off with cotton swabs. The cells were then fixed in $4 \%$ paraformaldehyde and stained with $0.5 \%$ crystal violet in methanol. An inverted microscope was used to count the number of invasive cells (XDS-800D, Shanghai Caikon Optical Instrument Co., Ltd., Shanghai, China) in five randomly selected visual fields. Each point was performed in triplicate with three independent experiments. 


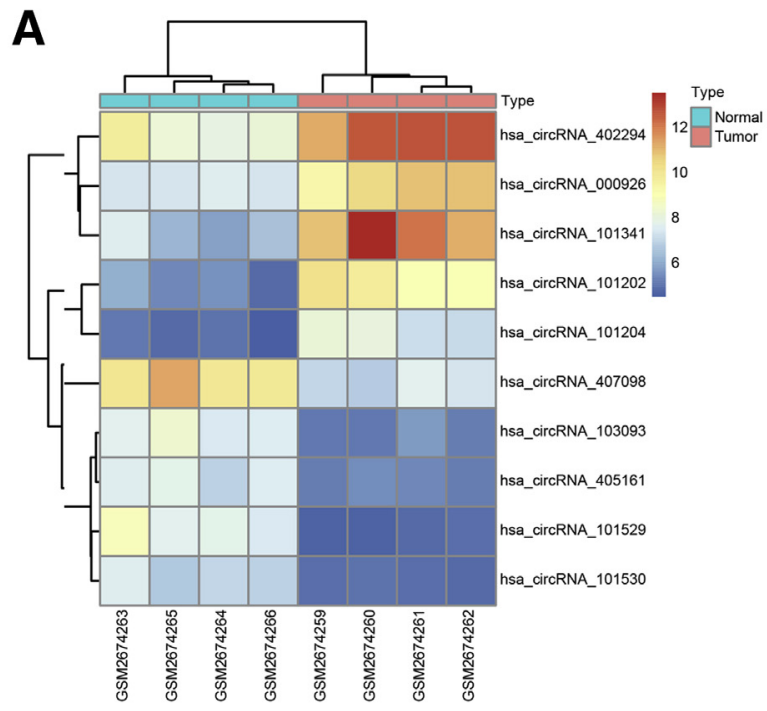

B

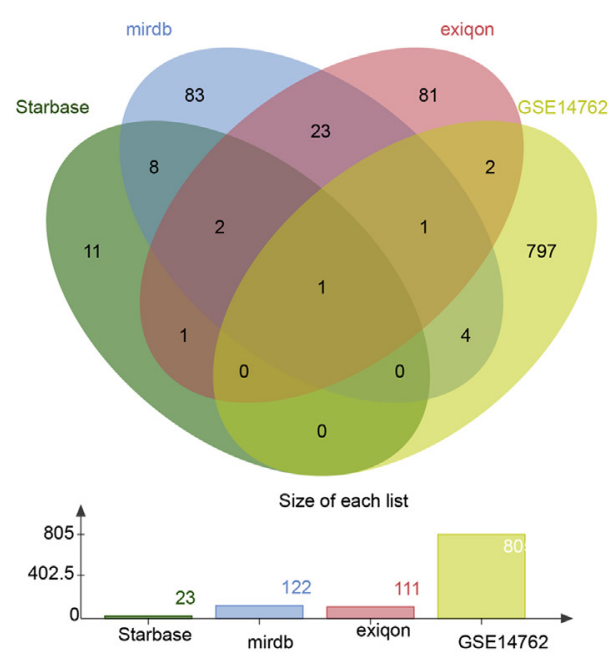

C

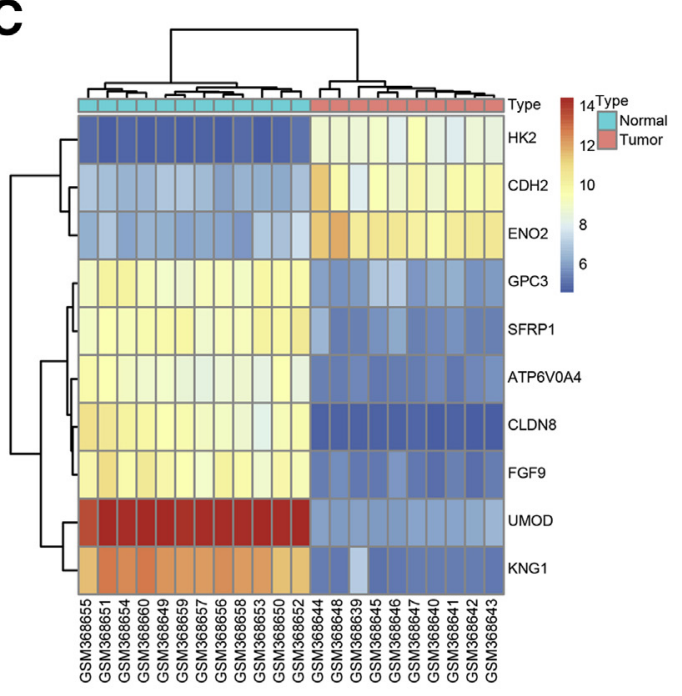

E

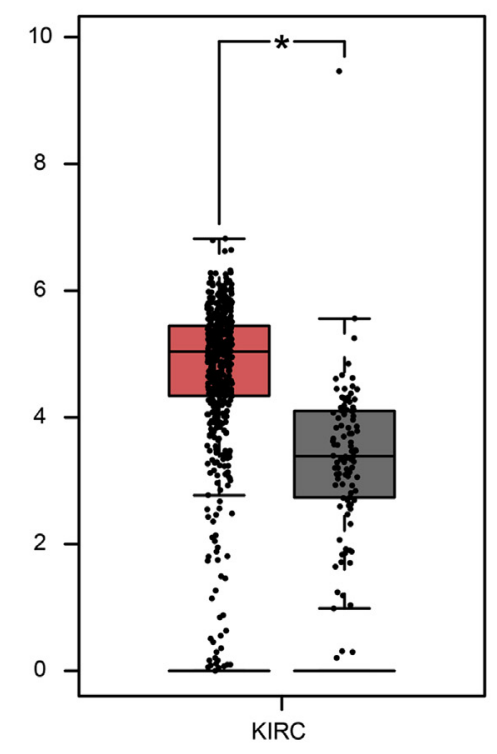

D
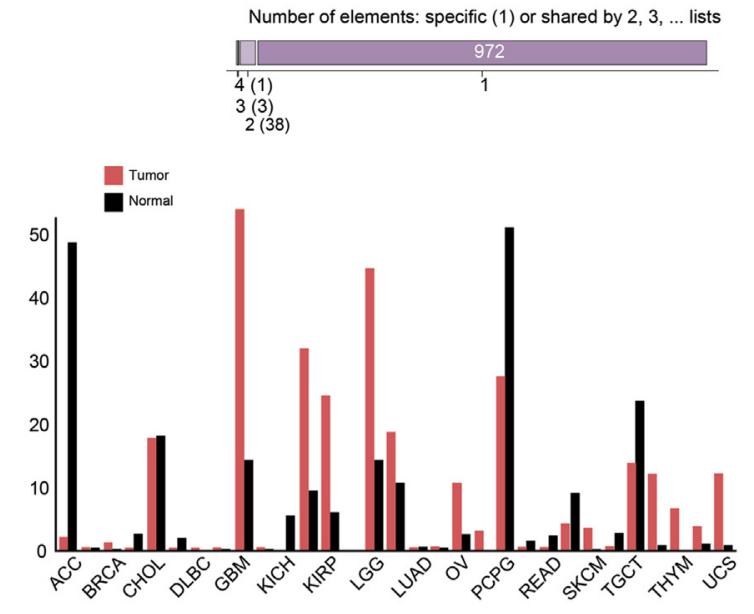

$\mathbf{F}$

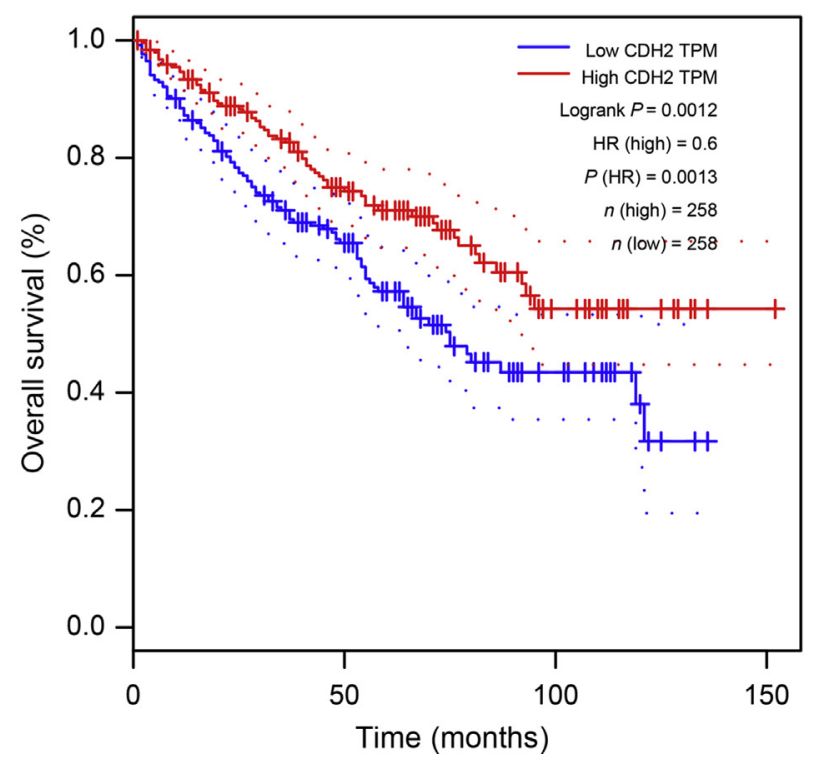




\section{Fluorescence in Situ Hybridization}

Fluorescence in situ hybridization (FISH) was used to determine the subcellular localization of circ_000926 in A498 cells according to instructions provided by the RibolncRNA FISH Probe Mix (Red, RiboBio Co., Ltd., Guangzhou, Guangdong, China). A498 cells were inoculated in 24-well plates at a density of $3 \times 10^{4}$ cells per well followed by 2-day culture. On reaching approximately $80 \%$ confluence, the cells were fixed in $1 \mathrm{~mL}$ of $4 \%$ paraformaldehyde for 10 minutes followed by treatment with $2 \mu \mathrm{g} / \mathrm{mL}$ of proteinase $\mathrm{K}$, glycine, and acetylation reagent. Cells were then incubated with $250 \mu \mathrm{L}$ of prehybridization solution and probed with $250 \mu \mathrm{L}$ of $300 \mathrm{ng} / \mathrm{mL}$ of hybridization solution containing probe. The cells were then stained with DAPI dye (1:800) for 5 minutes. After the cells were sealed with an antifluorescence quenching agent, five visual fields were randomly selected and captured under a fluorescence microscope (Olympus Optical Co., Ltd., Tokyo, Japan).

\section{Dual Luciferase Reporter Gene Assay}

The binding sites of miR-411 to circ_000926 and CDH2 were predicted using a biological prediction website. The full length of circ_000926 and the 3'-untranslated region $\left(3^{\prime}\right.$ UTR) region of $\mathrm{CDH} 2$ were cloned and amplified into a pmir GLO vector (E1330, Promega Corp., Madison, WI), which were named pcirc_000926-wild type (Wt) and pCDH2-Wt, respectively. The pcirc_000926-mutant (Mut) and pCDH2Mut plasmids were constructed by site-directed mutations in which the potential binding sites of miR-411 were mutated. pRL-TK vectors expressing Renilla luciferase (E2241, Promega Corp.) were used as internal reference. Either miR411 mimic or mimic NC was co-transfected into A498 cells with recombinant luciferase reporter plasmids, respectively. After 48 hours, the cells were collected and lysed. Luminescence at $560 \mathrm{~nm}$ (firefly relative luciferase activity) and $465 \mathrm{~nm}$ (Renilla relative luciferase activity) was measured according to the instructions of Dual Luciferase Reporter Gene Assay Kit (GM-040502A, Shanghai Qcbio Science \& Technologies Co., Ltd., Shanghai, China). The luciferase activity was expressed as the ratio of firefly relative luciferase activity to Renilla relative luciferase activity.

\section{RNA Pull-Down Assay}

Biotinylated probes were designed so that they could bind to the junction region of circ_000926, with oligonucleotide probes as controls. Approximately $1 \times 10^{7}$ cells were lysed with lysis buffer. The cell lysate was incubated with $3 \mu \mathrm{g}$ of biotinylated probes for 2 hours and antibiotic streptomycin magnetic beads (Life Technologies, Gaithersburg, MD) for 4 hours to pull-down the biotin-coupled RNA complexes. The magnetic beads were washed five times with lysis buffer, and the bound miRNAs in the pulled-down complex were extracted with Trizol reagent. Finally, miR-411 abundance was analyzed by RT-qPCR.

\section{Biotin-Coupled RNA Capture Assay}

Approximately $2 \times 10^{6}$ cells were transfected with $50 \mu \mathrm{mol} / \mathrm{L}$ biotinylated miR-411 mimic or NC (GenePharma, Shanghai, China) on reaching 50\% confluence and lysed 24 hours later. Cell lysate was then incubated with $50 \mu \mathrm{L}$ of streptavidin magnetic beads to pull down the biotin-conjugated RNA complex on a rotator for 4 hours at $10 \mathrm{r} / \mathrm{min}$. The beads were washed five times with lysis buffer, and the RNA interacting with miRNA was recovered by using Trizol LS reagent (Life Technologies). circ_000926 enrichment was detected by RTqPCR and agarose gel electrophoresis.

\section{Immunohistochemistry}

RCC tissues and adjacent normal tissues were fixed in $10 \%$ formalin, embedded, and cut into slices. The slices were dewaxed twice by xylene, dehydrated with gradient alcohol, and incubated with $3 \%$ hydrogen peroxide for 10 minutes to stop the endogenous catalase reaction. Next, 0.01 $\mathrm{mol} / \mathrm{L}$ citric acid buffer ( $\mathrm{pH}$ 6.0) was added to the slices for antigen retrieval. The slices were blocked with goat serum. Subsequently, the slices were incubated with monoclonal antibody against CDH2 (1:200, ab98952), E-cadherin (1:1000, ab239883), and vimentin (1:1000, ab92547). Phosphate-buffered saline replaced primary antibody as NC. After phosphate-buffered saline washing, the slices were incubated with goat anti-rabbit IgG (1:1000, ab6721) in a $37^{\circ} \mathrm{C}$ incubator for 30 minutes. The antibodies used were purchased from Abcam Inc. (Cambridge, MA). The slices were then developed with diaminobenzidine (Boster Biological Technology Co., Ltd., Wuhan, Hubei, China) for 2 minutes followed by counterstaining by hematoxylin (Nanjing Kaiji Bioengineering Co., Ltd., Nanjing, China). After dehydration and clearing, the slices were mounted using neutral balsam. Five random visual fields were selected and viewed under a microscope. Cells that appeared as brown- or yellow-stained cytoplasm were identified as positive cells. The positive rate of $\mathrm{CDH} 2$ protein equals the

Figure 1 Circular RNA 000926 (circ_000926) and cadherin 2 (CDH2) are up-regulated in renal cell carcinoma (RCC), and miR-411 is a candidate miRNA binding to circ_000926 and CDH2. A: The expression heat map of RCC-related differentially expressed circRNAs in the data set from the Gene Expression Omnibus (GEO) database (http://www.ncbi.nlm.nih.gov/geo; accession number GSE100186). B: Prediction of the target genes of miR-411. C: The expression heat map of RCC-related differentially expressed genes in the data set from the GEO database (accession number GSE14762). D: The expression of CDH2 in various cancers in The Cancer Genome Atlas (TCGA) database. E: The expression of CDH2 in 100 adjacent normal and 523 RCC tissues in TCGA database. F: Survival curve of patients with RCC with low and high $\mathrm{CDH} 2$ expression. ${ }^{\star} P<0.05$ versus normal tissues. HR, hazard ratio; KIRC, kidney renal clear cell carcinoma; TPM, transcripts per kilobase million. 

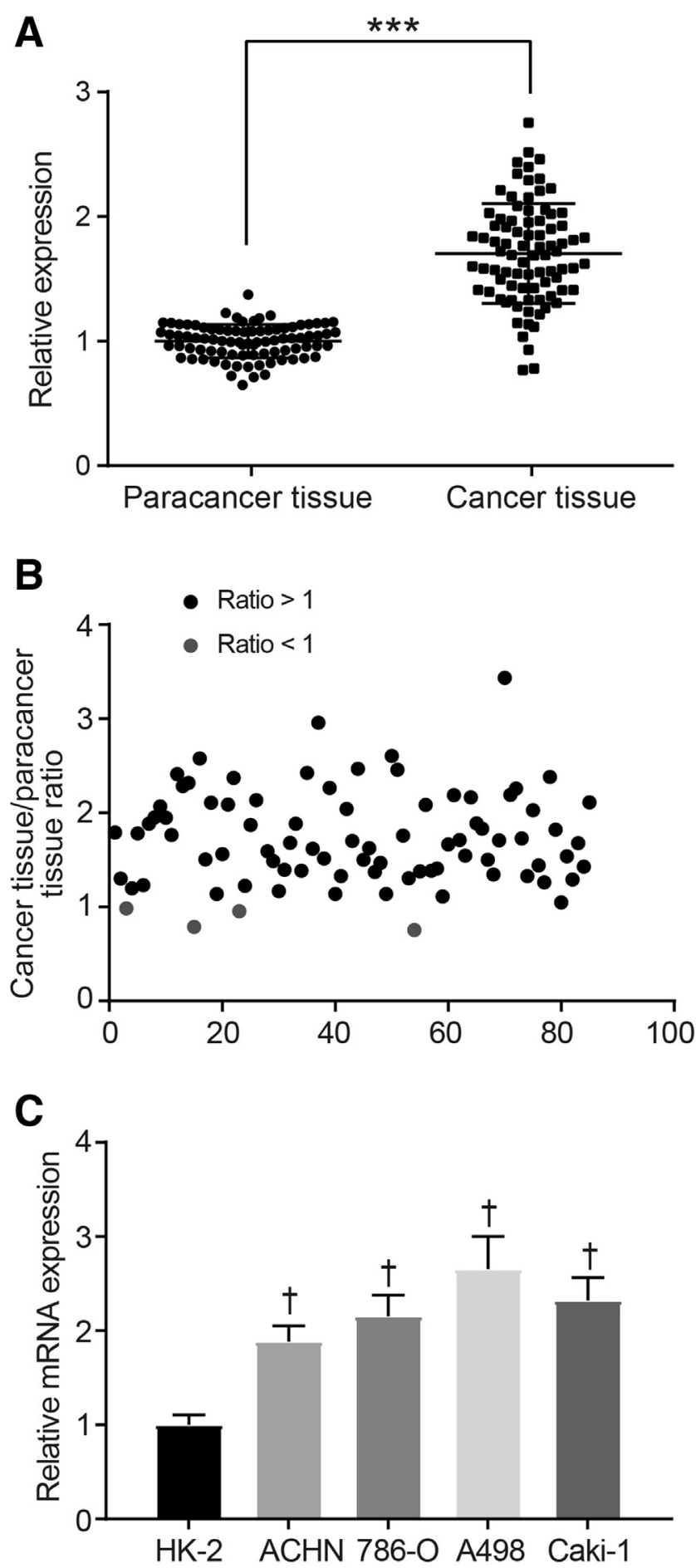

Figure 2 Renal cell carcinoma (RCC) exhibits a high expression of circular RNA 000926 (circ_000926). A: Expression of circ_000926 in adjacent normal and RCC tissues detected by real-time quantitative RT-PCR (RT-qPCR). B: The ratio of hsa_circ_000926 expression in RCC tissues to that in adjacent normal tissues. C: The expression of circ_000926 in cell lines 786-0, A498, Caki-1, and ACHN and normal renal HK-2 cell line detected by RT-qPCR. The experiment was performed in triplicate. Comparisons between two groups were performed using paired $t$-test and comparisons among multiple groups were assessed by one-way analysis of variance; Data are expressed as means \pm SD (C). $n=85$ (A and B). ${ }^{* * *} P<0.001$ versus adjacent normal tissues; ${ }^{\dagger} P<0.05$ versus normal renal HK-2 cell line. CDH2, cadherin 2; miR-411, miRNA-411. number of positive cells divided by the number of total cells.

Nude Mouse Xenograft Model of RCC

A total of $36 \mathrm{BALB} / \mathrm{c}-\mathrm{nu} / \mathrm{nu}$ mice (age, 4 to 5 weeks; weight, 22 to $24 \mathrm{~g} ; 18$ males and 18 females) were purchased from Vital River Laboratory Animal Technology Co., Ltd. (Beijing, China). A498 cells obtained during the in-logarithmic growth phase were transfected with si-circ_000926, miR-411 inhibitor, and/or oe-CDH2 to establish stably transfected cells. The stable cells were collected and resuspended into $5 \times 10^{7} / \mathrm{mL}$ cell suspension. The nude mice received subcutaneous inoculations of $0.2 \mathrm{~mL}$ of cell suspension using a 1$\mathrm{mL}$ syringe at their left axilla. After inoculation, the mice were housed in a laminar-flow hood under specific pathogenfree conditions. The maximum diameter $(\mathrm{L})$ and minimum diameter $(\mathrm{W})$ of the tumors were measured with a Vernier caliper every 7 days. The tumor volume $(\mathrm{V})$ was calculated by $=\mathrm{W}^{2} \times \mathrm{L} \times 0.52 .^{21}$

After 28 days, nude mice were euthanized, and transplanted tumors were excised. The tumors were fixed in $4 \%$ paraformaldehyde for 24 hours and then embedded with paraffin.

\section{Statistical Analysis}

SPSS software version 21.0 (IBM Corp., Armonk, NY) was used for statistical data analyses. Measurement data were presented as means \pm SD. The normal distribution and homogeneity of variances were examined. For the data obeying normal distribution and homogeneity of variances, intragroup comparison was conducted by paired $t$-test, and comparisons between two groups were performed using unpaired $t$-test or correlation test. Comparisons among multiple groups were assessed by one-way analysis of variance or repeated-measures analysis of variance. The experiment was repeated three times independently. $P<0.05$ was considered to be statistically significant.

\section{Results}

circ_000926 and CDH2 Are Highly Expressed in RCC and miR-411 Is a Candidate miRNA Binding to circ_000926 and $\mathrm{CDH} 2$

Differentially expressed circRNAs in RCC were initially screened based on RCC-related gene expression data sets downloaded from the GEO database (https://www.ncbi.nlm. nih.gov/geo; accession number GSE100186). Compared with adjacent normal tissues, circ_000926 levels were higher in RCC tissues (Figure 1A). The miRNAs interacting with circ_000926 were first predicted through the CircInteractome website (https://circinteractome.nia. nih.gov/miRNA_Target_Sites/mirna_target_sites.html, last 


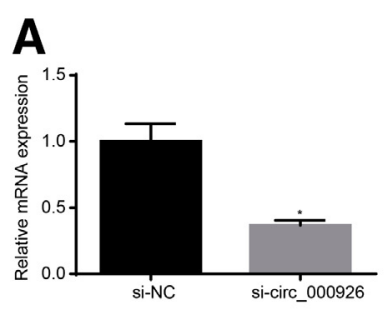

D
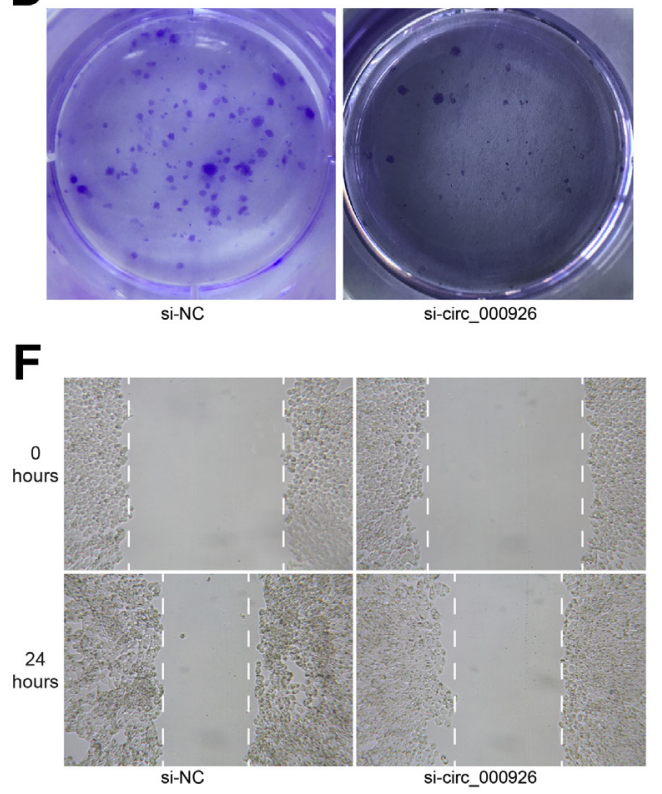

H

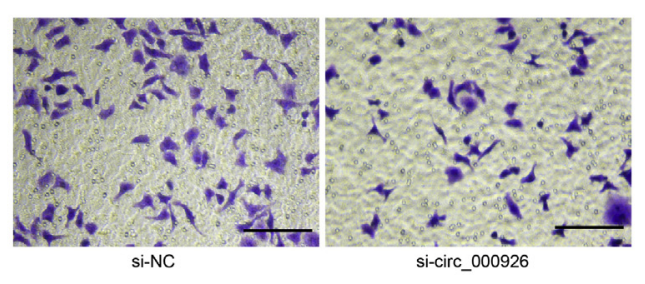

B

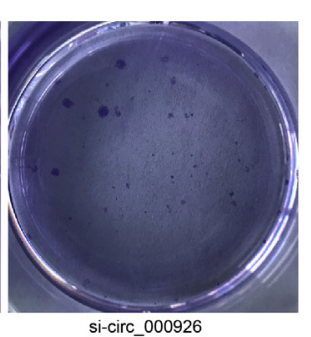

E

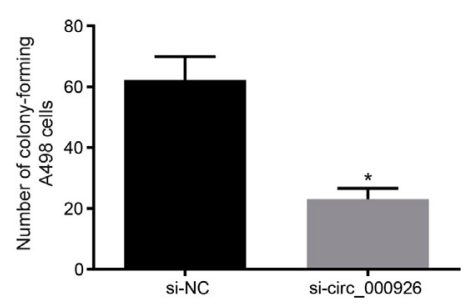

G
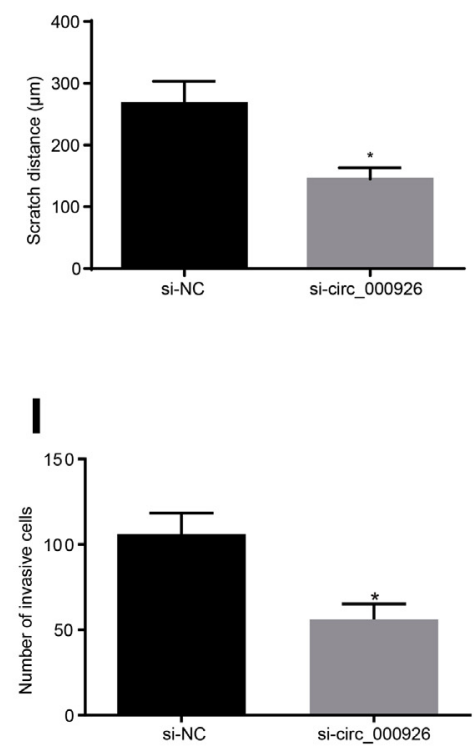

Figure 3 Inhibition of circular RNA 000926 (circ_000926) suppresses renal cell carcinoma (RCC) development. A: Expression of circ_000926 in A498 cells transfected with small interfering negative control (si-NC) or small interfering circ_000926 (si-circ_000926) measured by real-time quantitative RT-PCR (RT-qPCR). B: Expression of linear 000926 in A498 cells after intervention with si-NC or si-circ_000926 measured by RT-qPCR. C: The viability of A498 cells in response to silencing of circ_000926 assessed by the MTT assay. $\mathbf{D}$ and $\mathbf{E}$ : The number of colony-forming A498 cells in response to silencing of circ_000926 assessed by clonogenic assay. F and G: Migration of A498 cells in response to silencing of 000926 assessed by scratch test (the white dotted line in the figure indicates location of cell migration at the current time). $\mathbf{H}$ and $\mathbf{I}$ : Invasion of A498 cells after intervention with si-circ_000926 assessed by Transwell assay. Comparisons between two groups were performed using unpaired $t$-test, and comparisons among multiple groups were assessed by repeatedmeasures analysis of variance; the experiment was repeated three times independently. Data are expressed as means $\pm \mathrm{SD}$ ( $\mathbf{H}$ and $\mathbf{I}) .{ }^{*} P<0.05$ versus si-NC transfected cells. Original magnification: $\times 100(\mathbf{F}) ; \times 200(\mathbf{H})$. Scale bars $=50 \mu \mathrm{m}$ (H). accessed January 13, 2019), ${ }^{22}$ the results of which revealed that circ_000926 can bind to miR-411. The target genes of miR-411 were thus analyzed through Starbase (http:// starbase.sysu.edu.cn, last accessed January 13, 2019), mirdb (http://www.mirdb.org, last accessed January 13, 2019), and Exiqon of QIAGEN (Valencia, CA). ${ }^{23}$ The results of the intersection between candidate target genes and up-regulated genes in GSE14762 data sets identified that $\mathrm{CDH} 2$ was a target gene of miR-411 (Figure 1B) and was also highly expressed in RCC (Figure 1C). Analysis from the GEPIA website (http://gepia.cancer-pku.cn, last accessed January 13, 2019) suggested that $\mathrm{CDH} 2$ was upregulated in several cancers (Figure 1D) and found a significant increase in RCC (Figure 1E), the expression of which may be correlated with the prognosis of RCC (Figure 1F).

\section{circ_000926 Is Highly Expressed in RCC Tissues and} Cell Lines

To characterize the expression of circ_000926 in RCC, the expression of hsa_circ_000926 in the adjacent normal and RCC tissues was assessed. The expression of circ_000926 in RCC tissues was higher than that in adjacent normal tissues (Figure 2, A and B). Furthermore, circ_000926 expression in RCC cell lines 786-O, A498, Caki-1, and $\mathrm{ACHN}$ and the normal renal HK-2 cell line was analyzed, which found that the expression of circ_000926 was significantly higher in the four RCC cell lines compared with the normal renal HK-2 cell line, with the highest expression of circ_000926 observed in the A498 cell line (Figure 2C). Consequently, A498 cells were selected for the subsequent experiments. 

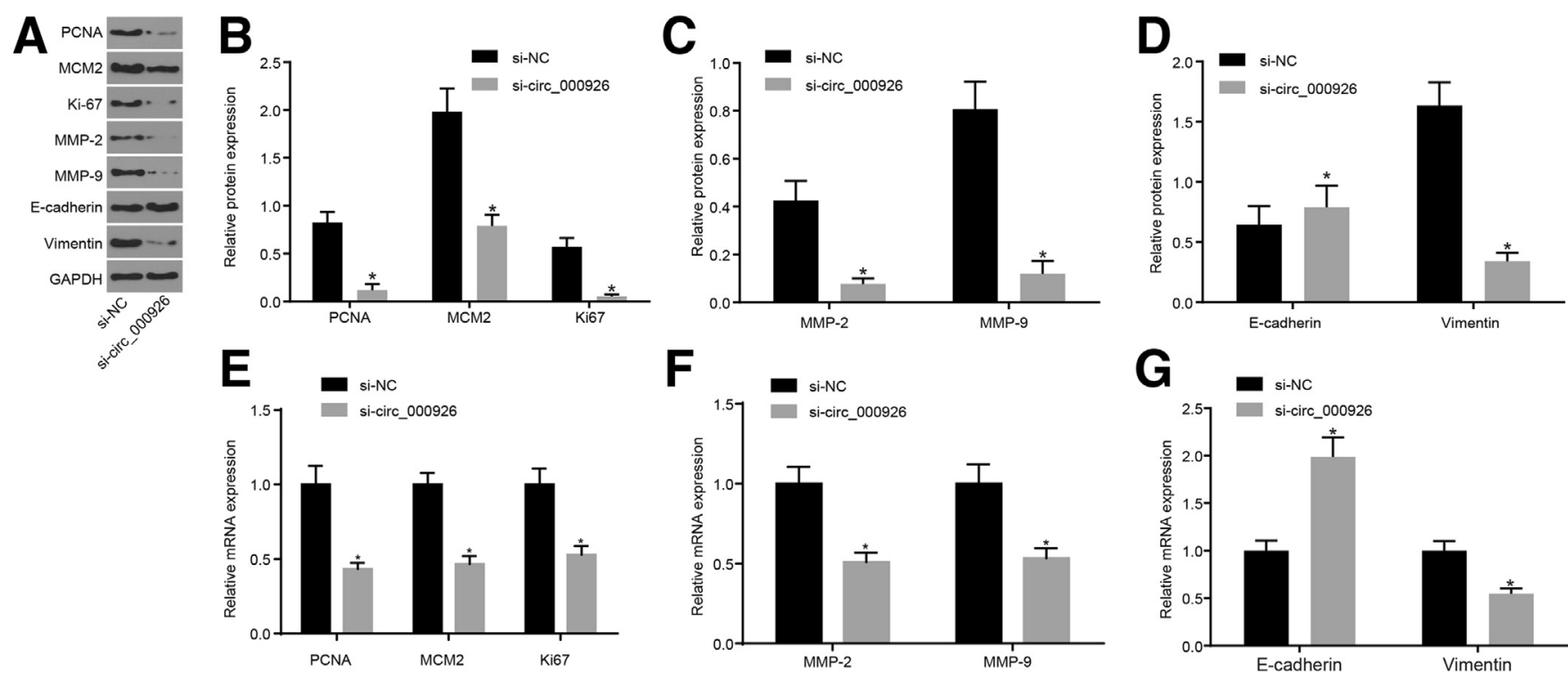

Figure 4 Inhibition of circular RNA 000926 (circ_000926) increases E-cadherin expression and decreases expression of proliferating cell nuclear antigen (PCNA), minichromosome maintenance complex component 2 (MCM2), Ki-67, matrix metalloproteinase (MMP)-2, MMP-9, and vimentin in renal cell carcinoma (RCC) cells. A-D: Western blot analysis of PCNA, MCM2, Ki-67, MMP-2, MMP-9, E-cadherin, and vimentin proteins in A498 cells introduced with small interfering circ_000926 (si-circ_000926). E-G: mRNA levels of the relevant genes in A498 cells introduced with si-circ_000926 measured by real-time quantitative RT-PCR. Comparisons between two groups were performed using unpaired $t$-test, and comparisons among multiple groups were assessed by repeated-measures analysis of variance. The experiment was repeated three times independently. Data are expressed as means \pm SD. ${ }^{*} P<0.05$ versus si-negative control (NC) transfected cells. GAPDH, glyceraldehyde-3-phosphate dehydrogenase.

\section{Down-Regulated circ_000926 Inhibits RCC Cell Invasion and Migration as Well as Proliferation and EMT}

To identify the potential role that circ_000926 played in RCC, circ_000926 was silenced in A498 cells using si-circ_000926. The knocking-down efficiency and specificity were analyzed by RT-qPCR, which revealed that circ_000926 expression in A498 cells significantly decreased with the delivery of si-circ_000926, whereas linear 000926 expression had no clear difference (Figure 3, A and B). A series of biological assays were performed to determine cellular functions after siRNA-mediated silencing of circ_000926 in A498 cells. The MTT assay found that cell viability in response to si-circ_000926 was markedly decreased (Figure 3C). In addition, in vitro clonogenic assays documented that the number of colony-forming cells in response to si-circ_000926 was noticeably less than that of those following si-NC treatment (Figure 3, D and E). The scratch test and Transwell assay found that the migration and invasion abilities of cells harboring si-circ_000926 were significantly decreased (Figure 3, F-I).

In addition, RT-qPCR and Western blot analysis were used to characterize the expression of genes related to proliferation (PCNA, MCM2, and Ki-67), migration (MMP-2 and MMP-9), and EMT (vimentin and E-cadherin) genes after sicirc_000926 was introduced into the A498 cells. The expression of PCNA, MCM2, Ki-67, MMP-2, MMP-9, and vimentin in cells transfected with si-circ_000926 decreased noticeably, whereas the expression of E-cadherin increased significantly
(Figure 4). Therefore, silencing of circ_000926 can suppress proliferation, colony formation, as well as migration and invasion abilities and reverse EMT induction in RCC cells.

\section{circ_000926 Functions as a Molecular Sponge of miR-411}

To determine whether circ_000926 could function as a competing endogenous RNA (ceRNA) to regulate miR411, the subcellular localization of circ_000926 and miR-411 was analyzed using FISH. Under merge conditions, blue (the nucleus), red (circ_000926), and green fluorescence (miR-411) were observed. This finding indicates that circ_000926 and miR-411 were co-localized in the cytoplasm (Figure 5A).

The binding sites between circ_000926 and miR-411 were predicted from biological prediction website. The results highlighted that there was a specific binding site between miR-411 and 3'-UTR of circ_000926 (Figure 5B). Dual luciferase reporter gene assay was also used to verify whether circ_000926 could bind to miR-411. Results indicated that the luciferase activity of p circ_000926-Wt in the miR-411 mimic group was significantly lower, whereas that of p circ_000926-Mut had no significant changes when compared with the NC group (Figure 5C), demonstrating that circ_000926 could competitively bind to miR-411.

The RNA pull-down assay found that circ_000926 was pulled down by specific circ_000926 probe (Figure 5D). The specific enrichment of miR-411 was 


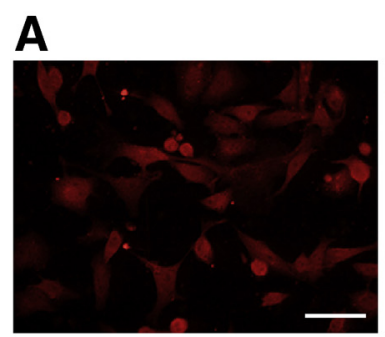

circ_000926

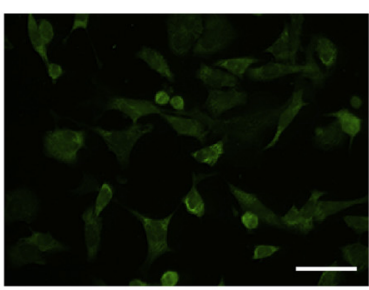

miR-411

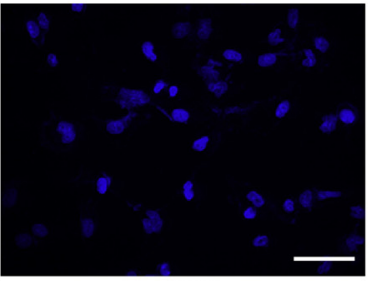

DAPI

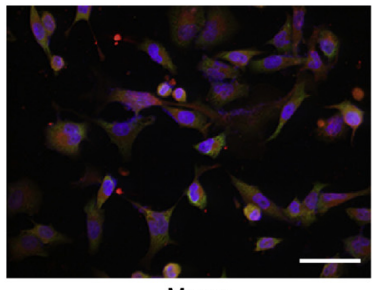

B
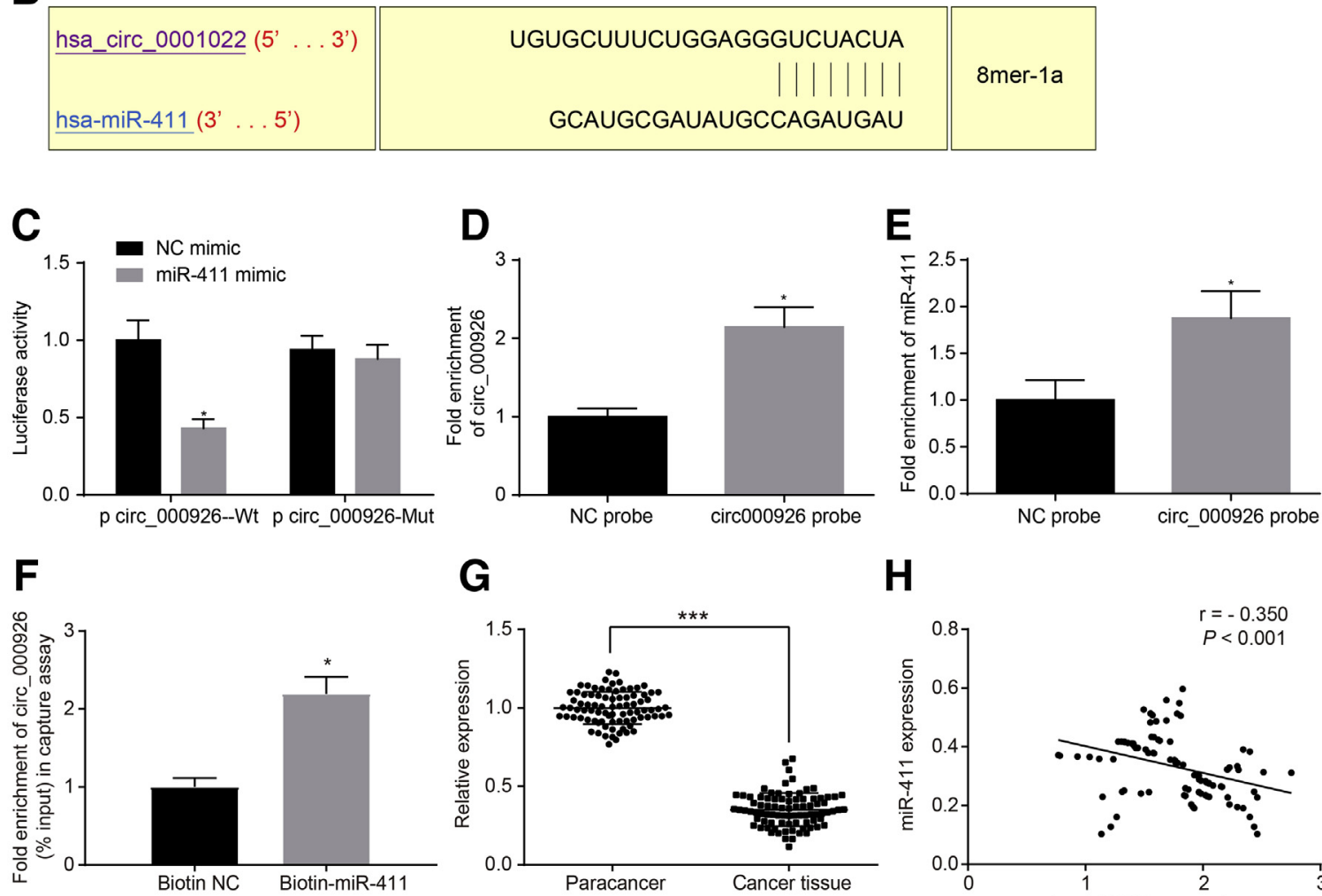

G

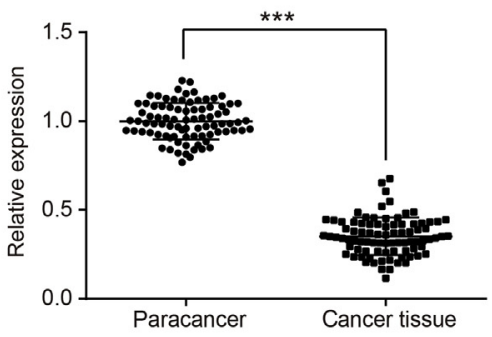

H

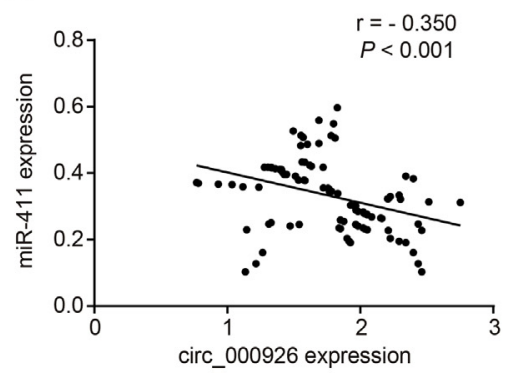

Figure 5 Circular RNA 000926 (circ_000926) acts as a molecular sponge of miRNA-411 (miR-411). A: Co-localization of circ_000926 and miR-411 in cells determined by fluorescence in situ hybridization; the red signal represents circ_000926, the green part represents miR-411, and the blue signal represents the nucleus. B: Predicted binding site of circ_000926 and miR-411 obtained from biological prediction website. C: The association between circ_000926 and miR411 verified by dual-luciferase reporter assay. D: Enrichment of circ_000926 by specific circ_000926 probe measured by real-time quantitative RT-PCR (RT-qPCR). E: Enrichment of miR-411 by specific circ_000926 probe measured by RT-qPCR. F: circ_000926 enrichment captured by biotin-coupled miR-411 measured by RT-qPCR. G: miR-411 expression in renal cell carcinoma (RCC) and adjacent normal tissues measured by RT-qPCR. H: Correlation analysis of expression of circ_000926 and miR-411 in RCC. Comparisons between two groups were performed using unpaired $t$-test or correlation analysis. The experiment was repeated three times independently. Data are expressed as means \pm SD. ${ }^{*} P<0.05,{ }^{* *} P<0.001$ versus negative control (NC) mimic, NC probe, or biotin NC groups. Original magnification, $\times 400$. Scale bar $=25 \mu \mathrm{m}$.

also detected in the beads pulled down by circ_000926 probe (Figure 5E), which manifested that circ_000926 could directly bind to miR-411. To validate the interaction between circ_000926 and miR-411, biotincoupled RNA capture assay was performed, which found circ_000926 enrichment in biotin-coupled miR-411 beads (Figure $5 \mathrm{~F}$ ), suggesting that miR-411 can bind to circ_000926.

In addition, the expression of miR-411 in RCC tissues was determined by RT-qPCR, which found that the expression of miR-411 in RCC tissues was noticeably lower than that in adjacent normal tissues (Figure 5G). The correlation analysis of both circ_000926 and miR-411 in RCC tissues further suggested that the expression of circ_000926 was negatively correlated with that of miR-411 (Figure 5H).

Overexpressed miR-411 Inhibits the Proliferation, Migration, and Invasion as Well as EMT in RCC Cells

Because circ_000926 can function as a miR-411 sponge, A498 cells were transfected with miR-411 mimic and 


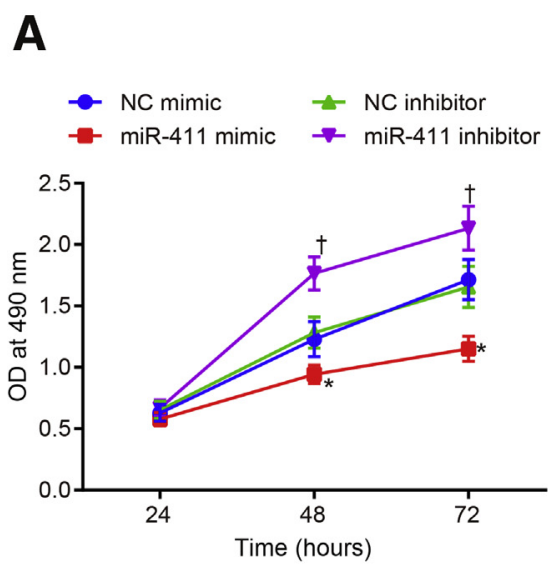

D

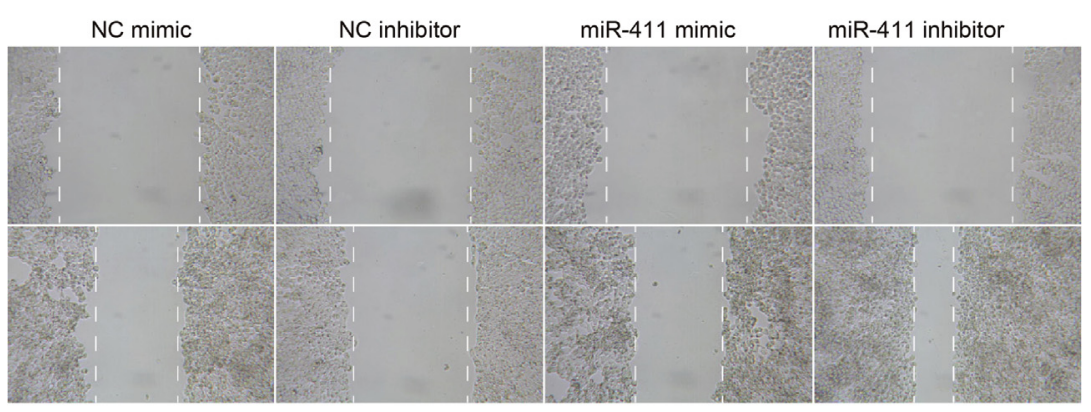

$\mathbf{F}$

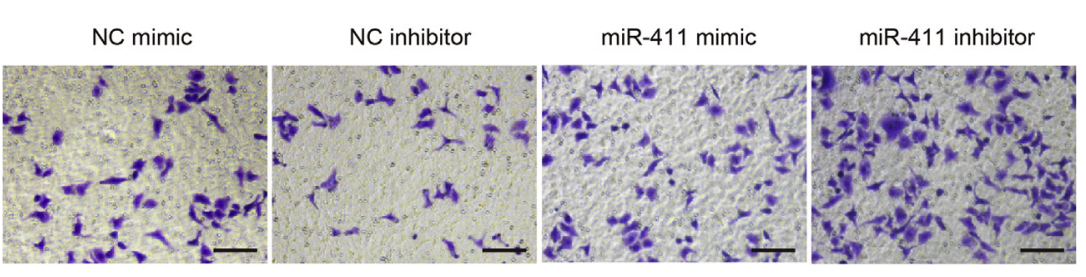

B

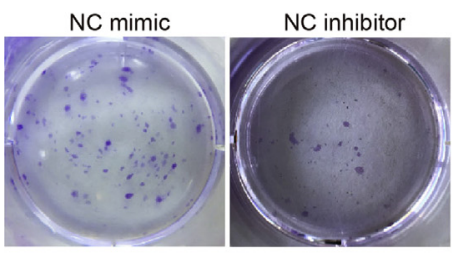

miR-411 mimic miR-411 inhibitor

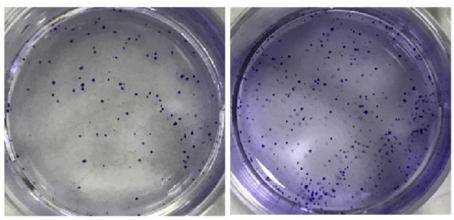

E

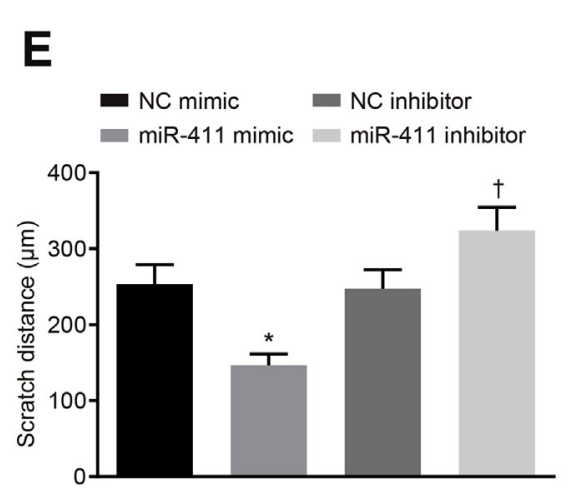
G $=\begin{aligned} & \text { NC mimic } \\ & \text { miR-411 mimic }=\text { minhibitor } \\ & \text { mi1 inhibitor }\end{aligned}$
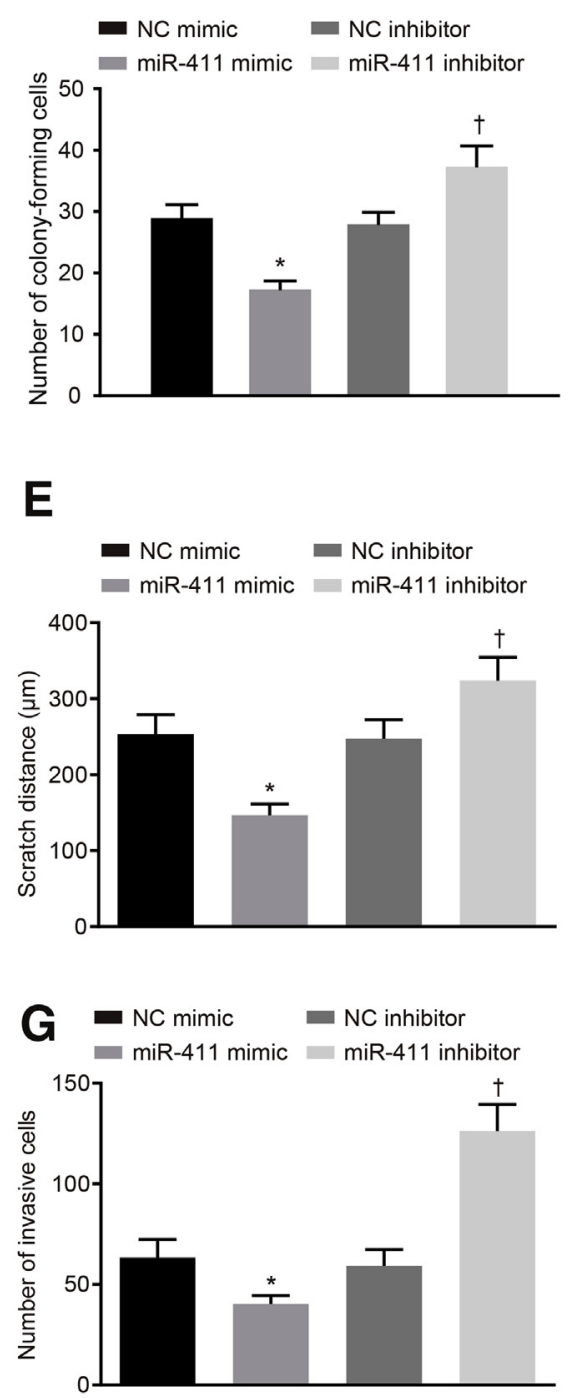

Figure 6 Overexpression of micro RNA-411 (miR-411) impedes renal cell carcinoma (RCC) cell proliferation, migration, and invasion. A: Cell viability in response to treatment with miR-411 mimic and miR-411 inhibitor assessed by MTT assay. B and C: The ability of cell colony formation in response to delivery of miR-411 mimic and miR-411 inhibitor assessed by clonogenic assay. D and E: Cell migration in response to transfection with miR-411 mimic and miR-411 inhibitor evaluated by scratch test (the white dotted line in the figure indicates location of cell migration at the current time). $\mathbf{F}$ and $\mathbf{G}$ : Cell invasion in response to transfection with miR-411 mimic and miR-411 inhibitor evaluated by Transwell assay. Comparisons among multiple groups were assessed by oneway analysis of variance or repeated-measures analysis of variance. The experiment was repeated three times independently. Data are expressed as means \pm SD. ${ }^{*} P<0.05$ versus cells transfected with miR-411 mimic negative control (NC); ${ }^{\dagger} P<0.05$ versus cells transfected with miR-411 inhibitor NC. Original magnification: $\times 100(\mathbf{D}) ; \times 200(\mathbf{F})$. Scale bar $=50 \mu \mathrm{m}(\mathbf{F})$.

miR-411 inhibitor, and different biological assays were conducted to evaluate cellular functions of miR-411 in RCC. MTT assay (Figure 6A) found that the proliferation of A498 cells was decreased by the miR-411 mimic and increased significantly by miR-411 inhibitor 48 and 72 hours after transfection.

Meanwhile, the results observed from clonogenic assays (Figure 6, B and C) revealed that the ability of colony formation markedly decreased in response to miR-411 mimic, which was noticeably enhanced by treatment with miR-411 inhibitor. The miR-411 inhibitor might have reversed the si-circ_000926-caused inhibition effect on the colony formation ability of A498 cells.

The scratch test and Transwell assay (Figure 6, D-G) found that, on miR-411 mimic treatment, A498 cell migration and invasion abilities were significantly attenuated. However, when miR-411 inhibitor was treated, significant enhancement of migration and invasion abilities was observed.

Subsequently, the expression of genes related to cell proliferation, migration (MMP-2 and MMP-9), and EMT (vimentin and E-cadherin) were measured by RT-qPCR and Western blot analysis. On miR-411 mimic treatment, the 

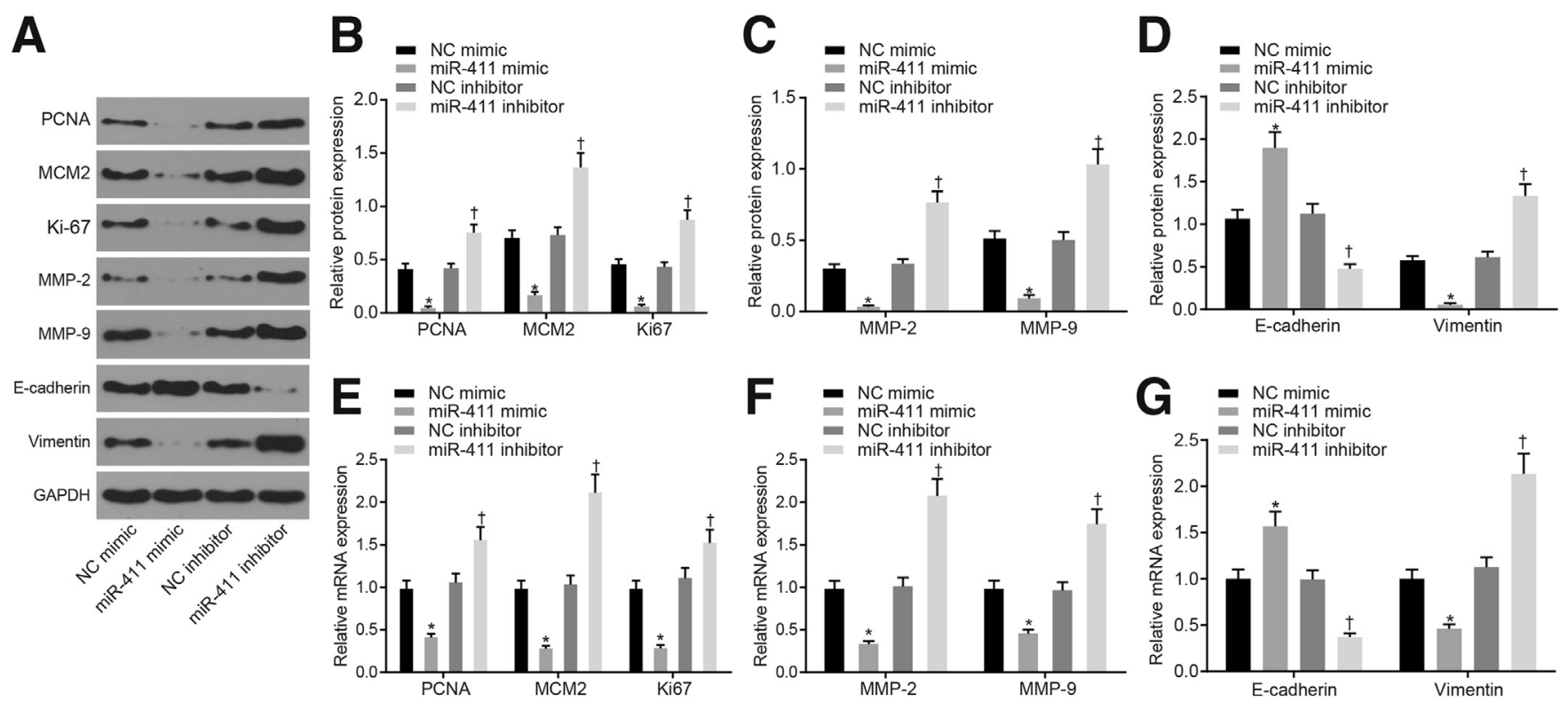

Figure 7 Inhibition of miRNA-411 (miR-411) increases E-cadherin expression and decreases expression of proliferating cell nuclear antigen (PCNA), minichromosome maintenance complex component 2 (MCM2), Ki-67, matrix metalloproteinase (MMP)-2, MMP-9, and vimentin in renal cell carcinoma (RCC) cells. A-D: Western blot analysis of PCNA, MCM2, Ki-67, MMP-2, MMP-9, E-cadherin, and vimentin proteins in cells treated with miR-411 mimic or miR-411 inhibitor. E-G: mRNA levels of the relevant genes in cells treated with miR-411 mimic or miR-411 inhibitor measured by real-time quantitative RT-PCR. Comparisons among multiple groups were assessed by one-way analysis of variance or repeated-measures analysis of variance. The experiment was repeated three times independently. Data are expressed as means $\pm \mathrm{SD}$. ${ }^{*} P<0.05$ versus cells transfected with miR-411 mimic negative control (NC); ${ }^{\dagger} P<0.05$ versus cells transfected with miR-411 inhibitor NC.

expression of PCNA, MCM2, Ki-67, MMP-2, MMP-9, and vimentin was significantly lower, whereas E-cadherin expression was significantly higher (Figure 7). The opposite effect was observed when treated with miR-411. Taken together, these results indicate that miR-411 overexpression can inhibit the proliferation, migration, invasion, and EMT in RCC cells.

\section{circ_000926 Facilitates RCC Cell Proliferation, Migration, and Invasion via Inhibition of miR-411}

To elucidate whether circ_000926 functioned in RCC progression via regulation of miR-411, A498 cells were transfected with si-NC plus NC inhibitor, si-circ_000926 plus NC inhibitor, and si-circ_000926 plus miR-411 inhibitor, followed by cell proliferation measurement using the MTT assay. A498 cell proliferation decreased markedly in response to sicirc_000926 plus NC inhibitor transfection (Figure 8A). Cell proliferation was also attenuated in response to si-circ_000926 plus miR-411 inhibitor transfection when compared with that on si-circ_000926 plus NC inhibitor transfection. This finding indicates how inhibited miR-411 leads to a significant reduction in the inhibitory effects of circ_000926 silencing on cell proliferation. Taken together, circ_000926 promoted RCC cell proliferation via down-regulating miR-411.

Meanwhile, the clonogenic assay was applied to further explore the results observed from the MTT assay. Colony formation ability was significantly lower in response to sicirc_000926 plus NC inhibitor (Figure 8, B and C).
However, the ability of colony formation was noticeably enhanced by transfection with si-circ_000926 plus miR-411 inhibitor compared with si-circ_000926 plus NC inhibitor transfection. This finding indicated that inhibited miR-411 can significantly reverse the inhibitory effects of circ_000926 silencing on the ability of colony formation. Overall, circ_000926 stimulated RCC cell proliferation via down-regulating miR-411.

The scratch test and Transwell assay were performed to evaluate the possible effects of circ_000926 on RCC cell migration and invasion. On si-circ_000926 plus NC inhibitor treatment, A498 cell migration and invasion were significantly decreased (Figure $8, \mathrm{D}-\mathrm{G}$ ). The treatment of si-circ_000926 plus miR-411 inhibitor resulted in significant enhancement of migration and invasion abilities compared with si-circ_000926 plus NC inhibitor treatment. Therefore, these results suggest that inhibited miR-411 can significantly disrupt the inhibitory effects of circ_000926 silencing on the ability of cell migration and invasion abilities. Taken together, circ_000926 promoted RCC cell migration and invasion via down-regulating miR-411.

circ_000926 Acts as a ceRNA for miR-411 to Increase CDH2 Expression and Facilitates RCC Cell Proliferation, Migration, Invasion, and EMT

Bioinformatics analysis was conducted to clarify the downstream target genes of miR-411. Results revealed the existence of a binding site between miR-411 and $\mathrm{CDH} 2$ 

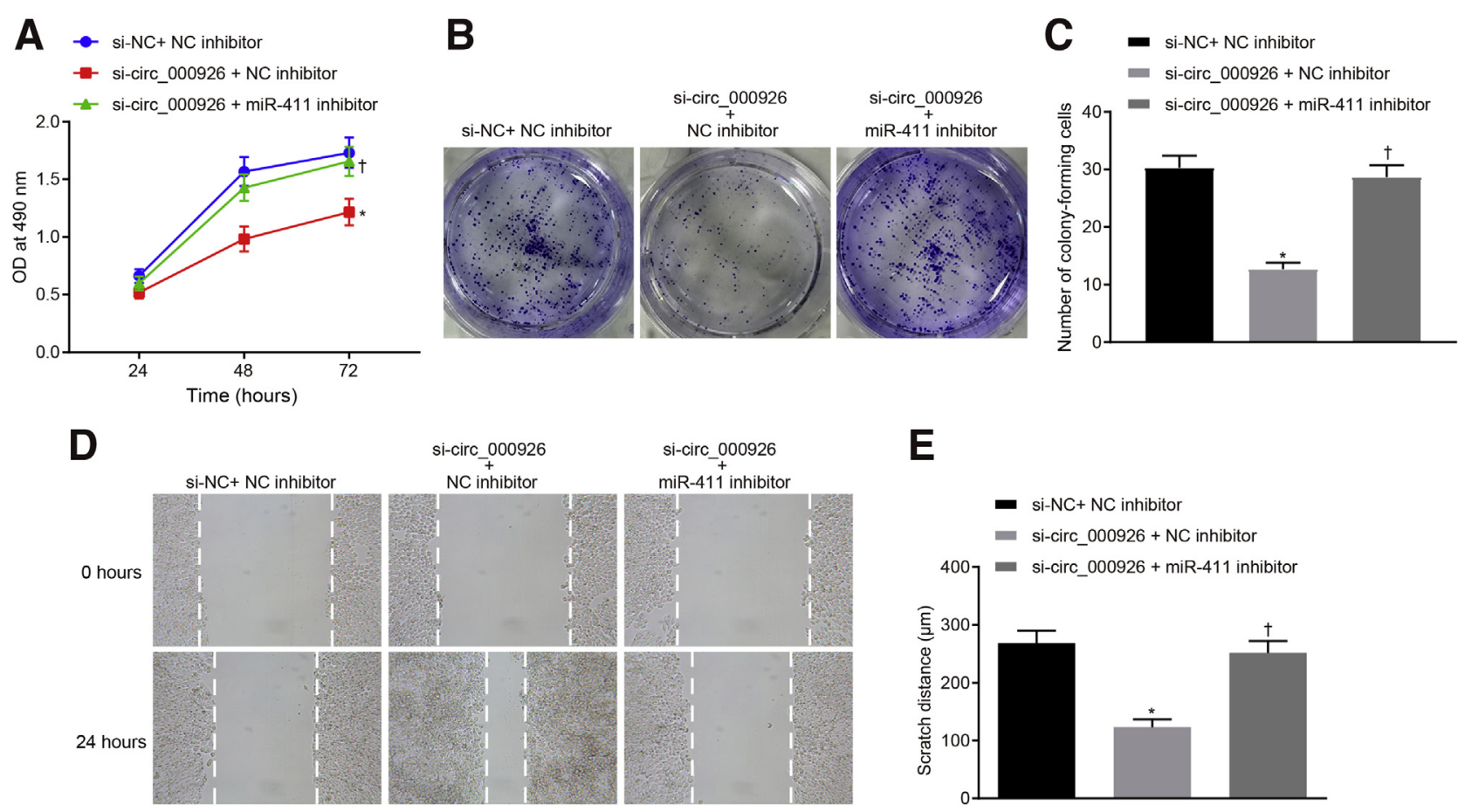

E
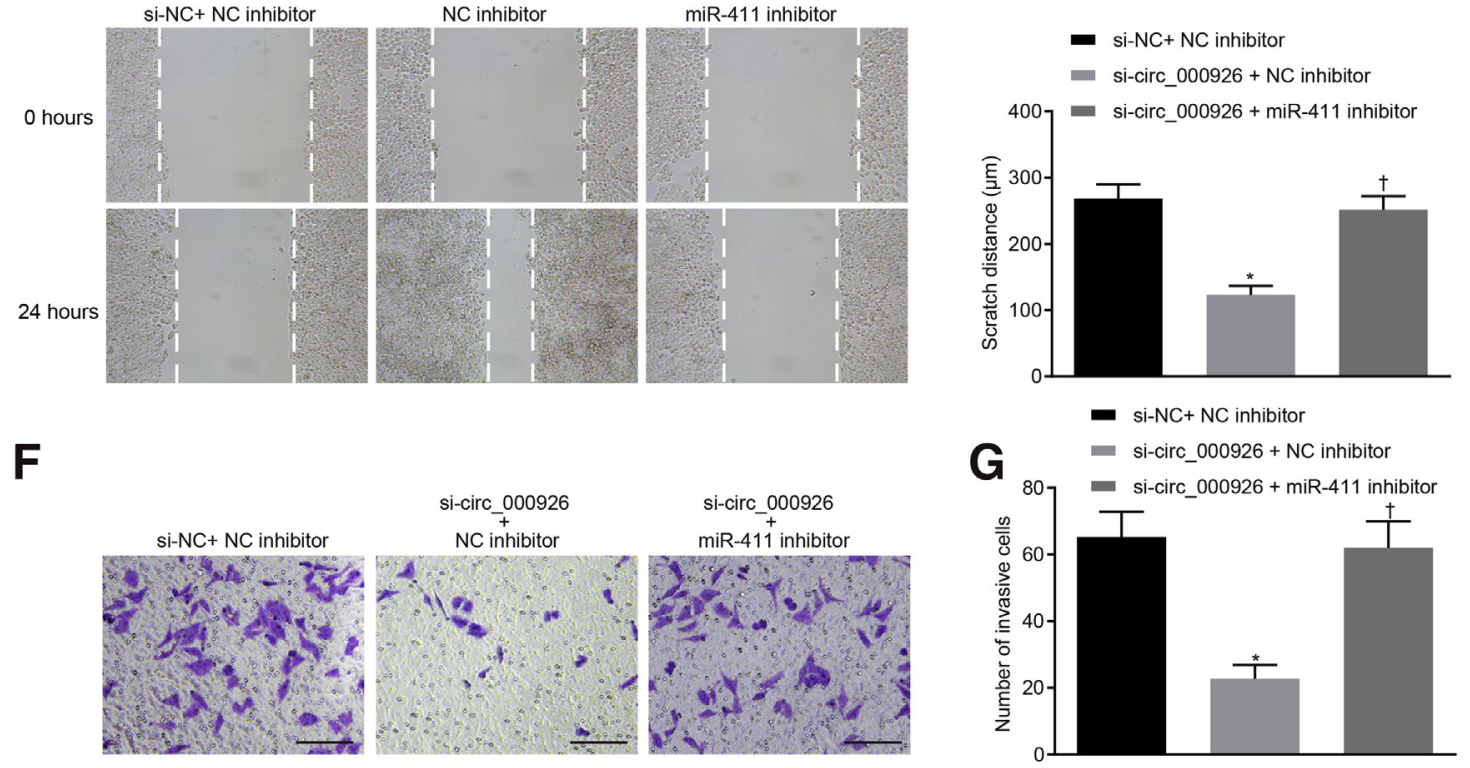

Figure 8 Circular RNA 000926 (circ_000926) induces renal cell carcinoma (RCC) cell proliferation, migration, and invasion via inhibition of miRNA-411 (miR411). A: Cell viability in response to small interfering circ_000926 (si-circ_000926) and miR-411 inhibitor treatment assessed by the MTT assay. B and C: The ability of cell colony formation in response to si-circ_000926 and miR-411 inhibitor treatment assessed by clonogenic assay. D and E: Migration of cells transfected with si-circ_000926 and miR-411 inhibitor evaluated by scratch test (the white dotted line in the figure indicates location of cell migration at the current time). F and G: Cell invasion in response to si-circ_000926 and miR-411 inhibitor treatment evaluated by Transwell assay. Comparisons among multiple groups were assessed by one-way analysis of variance or repeated-measures analysis of variance. The experiment was repeated three times independently. Data are expressed as means \pm SD. ${ }^{*} P<0.05$ versus si-negative control (si-NC) plus NC inhibitor transfected cells; ${ }^{\dagger} P<0.05$ versus si-circ_000926 plus NC inhibitor transfected cells. Original magnification: $\times 100$ (D); $\times 200$ (G). Scale bar $=50 \mu \mathrm{m}(\mathbf{G})$. CDH2, cadherin 2; oe, overexpression.

(Figure 9A). Dual luciferase reporter gene assay results indicated that the luciferase activity of $\mathrm{CDH} 2-\mathrm{Wt} 3^{\prime}$-UTR was significantly lower after co-transfection of miR-411 mimic, whereas that of CDH2-mut $3^{\prime}$-UTR was not affected by co-transfection of miR-411 mimic (Figure 9B). The expression of $\mathrm{CDH} 2$ was further characterized in $\mathrm{RCC}$ tissues by RT-qPCR, which found that $\mathrm{CDH} 2$ expression in RCC tissues was remarkably higher than that of adjacent normal tissues (Figure 9C). Correlation analysis further confirmed that the expression of circ_000926 was positively correlated with that of $\mathrm{CDH} 2$, whereas the expression of miR-411 was negatively correlated with that of $\mathrm{CDH} 2$ (Figure 9, D and E).

To further explore the potential effects of $\mathrm{CDH} 2$ on the proliferation of RCC cells, MTT assays were performed in A498 cells that were treated with si-NC plus oe-CDH2 and si-circ_000926, si-NC plus oe-CDH2 and miR-411 mimic, or si-NC plus oe-CDH2 alone. No noticeable changes in the OD value were observed at 24 hours after treatment. However, the proliferation of cells transfected with si$\mathrm{NC}$ plus oe-CDH2 at 48 and 72 hours after treatment was significantly higher than that of cells transfected with si$\mathrm{NC}$ plus oe-NC. On the other hand, the proliferation of cells transfected with si-circ_000926 plus oe-CDH2 at 48 and 72 hours after treatment was significantly lower than those subjected to si-NC plus oe-CDH2 transfection. Overall, $\mathrm{CDH} 2$ overexpression can reverse the inhibitory effects of si-circ_000926 on cell proliferation (Figure 10A).

Meanwhile, the effects of $\mathrm{CDH} 2$ on the ability of colony formation of A498 cells were tested using the clonogenic assay. The ability of colony formation of si-NC plus oe-CDH2 transfected cells was significantly higher than that of si- 


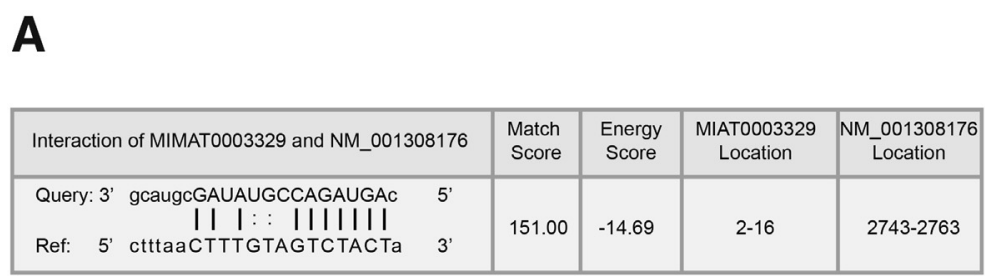

C

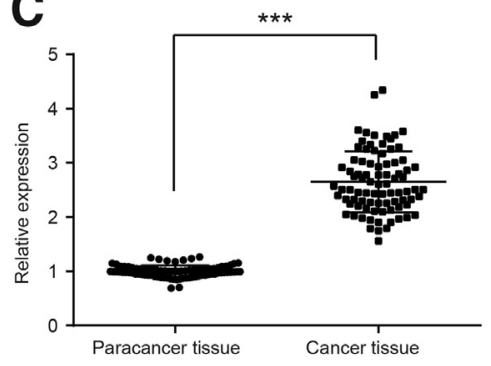

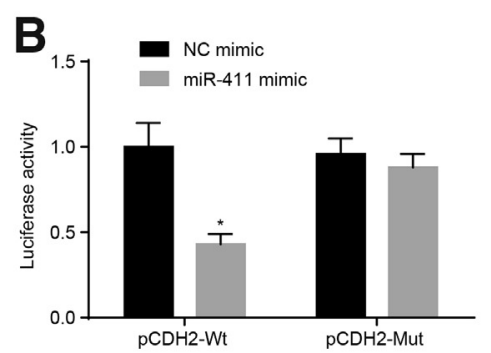

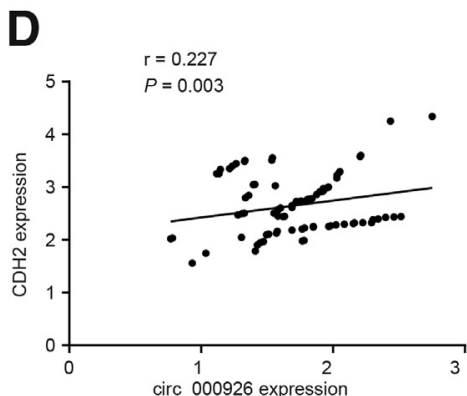

$E$

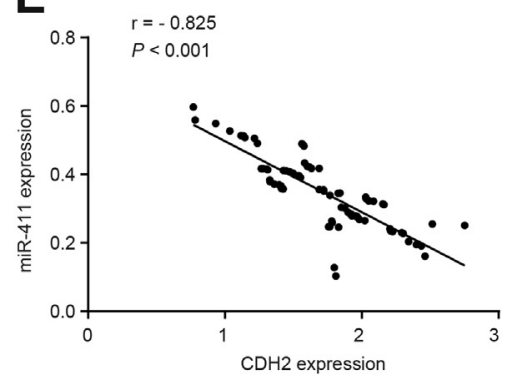

Figure 9 Circular RNA 000926 (circ_000926) functions as a competing endogenous RNA (ceRNA) for miRNA-411 (miR-411) to increase cadherin 2 (CDH2) levels, thereby mediating renal cell carcinoma (RCC) progression. A: Predicted binding site of miR-411 to $\mathrm{CDH} 23^{\prime}$-untranslated region analyzed by biological prediction website. B: The association between $\mathrm{CDH} 2$ and miR-411 verified by dual-luciferase reporter assay. C: $\mathrm{CDH} 2$ expression in RCC and adjacent normal tissues measured by real-time quantitative RT-PCR. D: Correlation analysis of expression of circ_000926 and CDH2. E: Correlation analysis of expression of miR411 and $\mathrm{CDH} 2$. Comparisons between two groups were performed using unpaired $t$-test or correlation analysis. Comparisons among multiple groups were assessed by one-way analysis of variance or repeated-measures analysis of variance. The experiment was repeated three times independently. Data are expressed as means $\pm \mathrm{SD} .{ }^{*} P<0.05,{ }^{* *} P<0.001$ versus negative control (NC) mimic-transfected cells or adjacent normal tissues.

$\mathrm{NC}$ plus oe-NC transfected cells, whereas that of si-circ_000926 plus oe-CDH2 transfected cells was significantly lower than that of si-NC plus oe-CDH2 transfected cells (Figure 10, B and C). Overall, $\mathrm{CDH} 2$ overexpression could reverse the suppressive effects of circ_000926 silencing on colony formation of RCC cells.

The scratch test and Transwell assay were conducted to evaluate the controlling of $\mathrm{CDH} 2$ in the migration and invasion abilities of A498 cells. Transfection of si-NC plus oe-CDH2 was observed to remarkably enhance migration and invasion abilities of cells, whereas transfection of si-circ_000926 plus oe-CDH2 led to significantly attenuated migration and invasion abilities of cells compared with si-NC plus oe-CDH2 transfection. Therefore, $\mathrm{CDH} 2$ overexpression rescued the migration- and invasion-inhibiting effects of circ_000926 silencing (Figure 10, D-G).

Subsequently, the expression of cell proliferation-, migration-, and EMT-related genes was determined using RT-qPCR and Western blot analysis. On si-NC plus oe$\mathrm{CDH} 2$ transfection, the expression of PCNA, MCM2, Ki67, MMP-2, MMP-9, and vimentin was significantly higher, whereas E-cadherin expression was remarkably lower (Figure 10, H-N). However, the expression of PCNA, MCM2, Ki-67, MMP-2, MMP-9, and vimentin was significantly decreased, whereas E-cadherin expression was remarkably elevated in response to si-circ_000926 plus oe$\mathrm{CDH} 2$ transfection when compared with si-NC plus oe$\mathrm{CDH} 2$ transfection. Overall, $\mathrm{CDH} 2$ overexpression reversed the effects of circ_000926 silencing on the expression of the aforementioned genes. Therefore, circ_000926 can serve as a ceRNA for miR-411 to increase CDH2 in RCC by which circ_000926 mediates the developmental process of RCC.

\section{Up-Regulated miR-411 Represses RCC Cell Proliferation, Migration, and Invasion via Inhibition of $\mathrm{CDH} 2$}

To investigate whether miR-411 affected RCC cell proliferation, migration, and invasion via regulation of $\mathrm{CDH} 2, \mathrm{~A} 498$ cells were transfected with $\mathrm{NC}$ mimic plus oe-NC, miR-411 mimic plus oe-NC, and miR-411 mimic plus oe-CDH2, followed by cell proliferation measurement using the MTT assay. Cell proliferation decreased significantly in response to miR-411 mimic plus oe-NC transfection (Figure 11A). However, the opposite effect was observed in response to miR-411 mimic plus oe-CDH2 transfection when compared with those transfected with the miR-411 mimic plus oe-NC. This finding indicates that miR-411 exerts effects on cell proliferation via regulation of $\mathrm{CDH} 2$.

The results obtained from the clonogenic assay (Figure 11, B and C) indicate that the ability of colony formation significantly decreased in response to miR-411 mimic plus oe-NC transfection. However, an increase was seen in the colony formation after transfection with miR411 mimic plus oe-CDH2 compared with miR-411 mimic plus oe-NC transfection. This finding indicates that 
miR-411 exerts effects on cell proliferation by regulating $\mathrm{CDH} 2$.

The scratch test and Transwell assay found that miR-411 mimic plus oe-NC transfection leads to significantly increased A498 cell migration and invasion. The transfection of miR-411 mimic plus oe-CDH2 resulted in a reduction of migration and invasion abilities compared with miR-411 mimic plus oe-NC transfection (Figure 11, D-G). The aforementioned findings suggested that miR-411 functioned in cell migration and invasion via regulation of $\mathrm{CDH} 2$.

\section{Down-Regulation of circ_000926 Inhibits Xenograft Tumor Growth of Nude Mice}

To validate the effects of circ_000926 on RCC progression, an in vivo xenograft nude mouse model was established by injecting A498 cells that expresses miR-411 inhibitor or
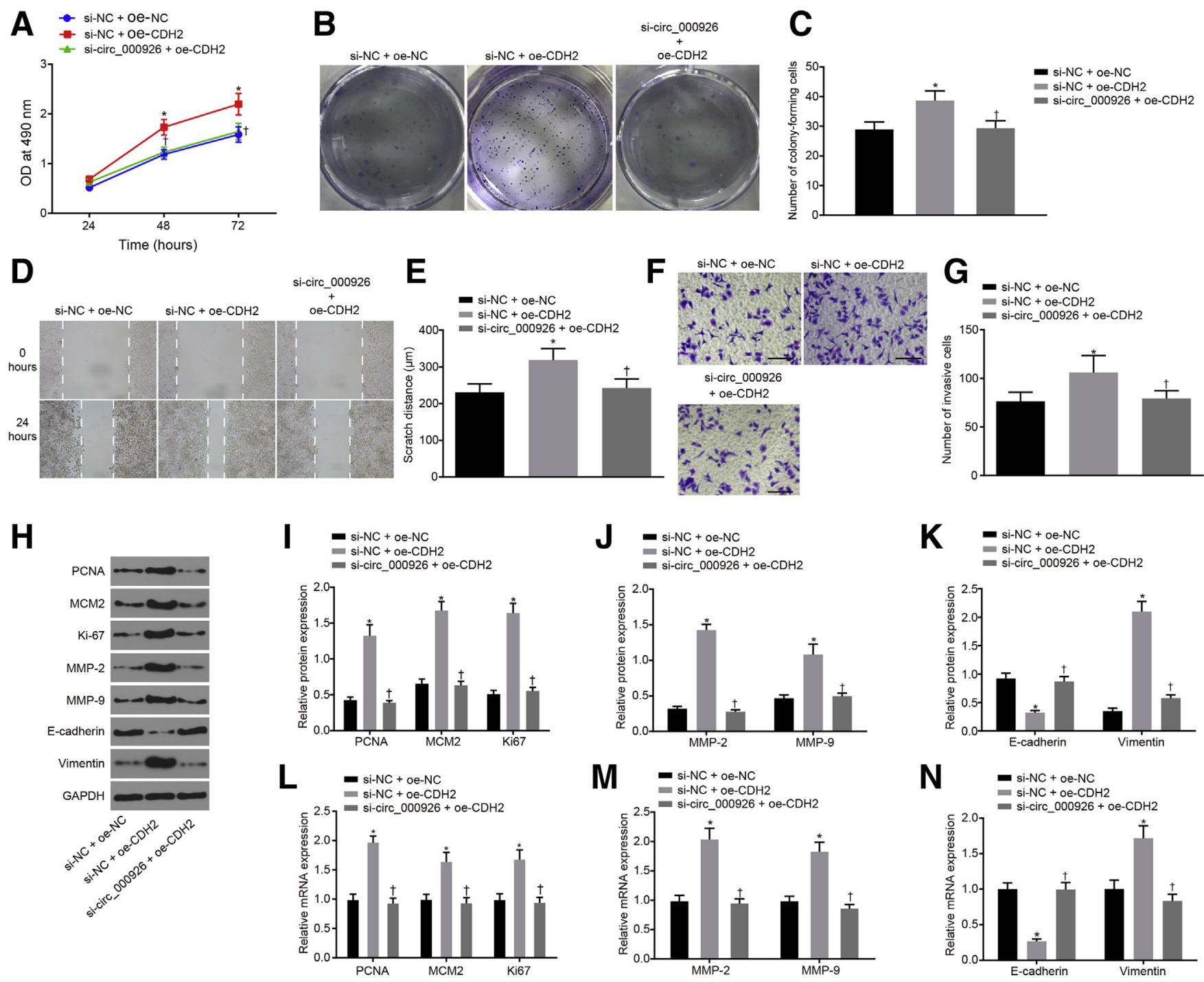

Figure 10 Circular RNA 000926 (circ_000926) elevates cadherin 2 (CDH2) expression to promote renal cell carcinoma (RCC) cell migration and invasion. A: Cell viability in response to overexpression (oe)-CDH2 alone or small interfering circ_000926 (si-circ_000926) and oe-CDH2 treatment assessed by the MTT assay. B and C: The ability of cell colony formation in response to oe-CDH2 alone or si-circ_000926 and oe-CDH2 treatment assessed by clonogenic assay. D and E: Migration of cells transfected with oe-CDH2 alone or si-circ_000926 and oe-CDH2 evaluated by scratch test (the white dotted line in the figure indicates location of cell migration at the current time). $\mathbf{F}$ and $\mathbf{G}$ : Cell invasion in response to oe-CDH2 alone or si-circ_000926 and oe-CDH2 treatment evaluated by Transwell assay. H-K: Western blot analysis of proliferating cell nuclear antigen (PCNA), minichromosome maintenance complex component 2 (MCM2), Ki-67, matrix metalloproteinase (MMP)-2, MMP-9, E-cadherin, and vimentin proteins in cells transfected with oe-CDH2 alone, si-circ_000926 and oeCDH2, or miR-411 mimic and oe-CDH2. L-N: mRNA levels of the relevant genes in cells transfected with oe-CDH2 alone, si-circ_000926 and oe-CDH2, or miR411 mimic and oe-CDH2 measured by real-time quantitative RT-PCR. Comparisons between two groups were performed using the unpaired $t$-test or correlation analysis. Comparisons among multiple groups were assessed by one-way analysis of variance or repeated-measures analysis of variance. The experiment was repeated three times independently. Data are expressed as means \pm SD. ${ }^{*} P<0.05$ versus small interfering negative control (si-NC) plus oe-NC transfected cells, ${ }^{\dagger} P<0.05$ versus si-circ_000926 plus oe-NC transfected cells. Original magnification: $\times 100$ (D); $\times 200(\mathbf{F})$. Scale bar $=50 \mu \mathrm{m}(\mathbf{F})$. GAPDH, glyceraldehyde-3-phosphate dehydrogenase; Mut, mutant. 


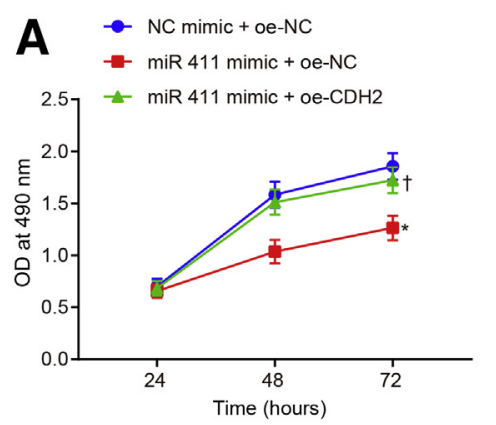

D

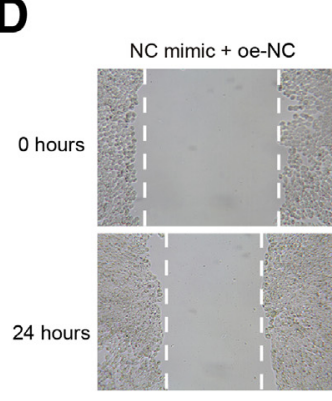

$\mathbf{F}$

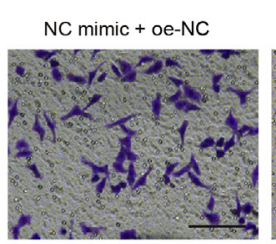

miR 411 mimic
B

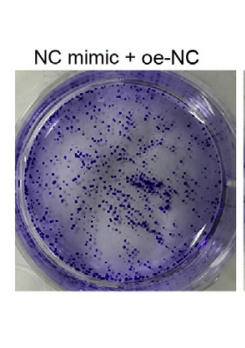

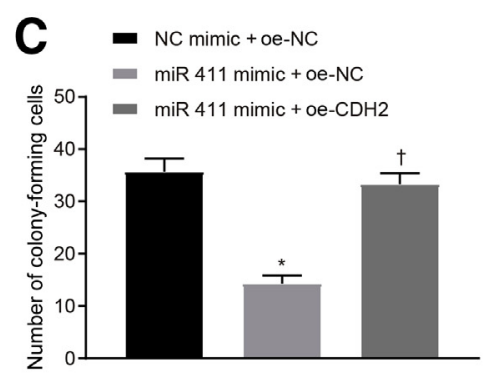

$\mathbf{E}$

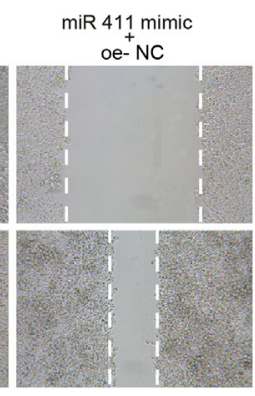

$\mathrm{miR} 411$ mimic oe- $\stackrel{+}{\mathrm{C}} \mathrm{DH} 2$
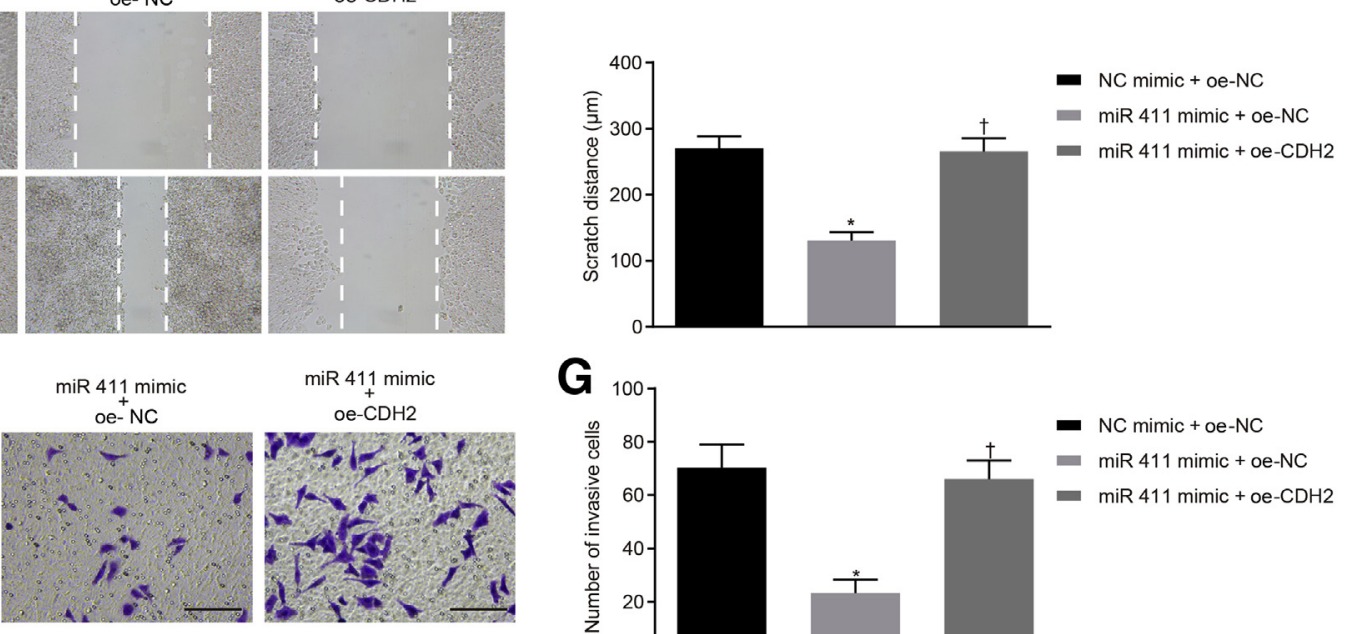

miR 411 mimic oe- $\stackrel{+}{\mathrm{CDH}} 2$

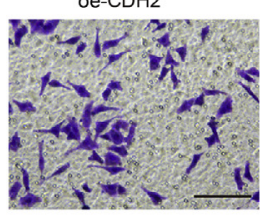

G

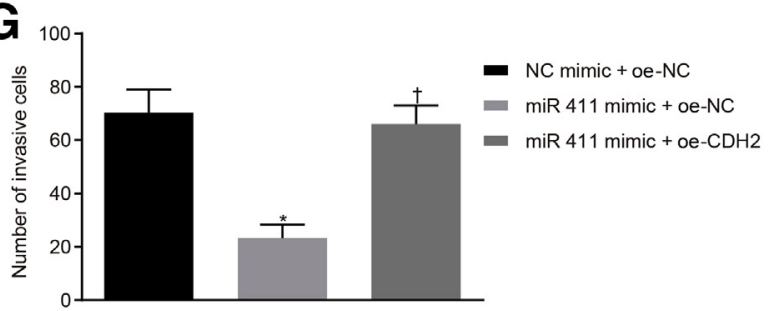

Figure 11 Up-regulated miRNA-411 (miR-411) impedes renal cell carcinoma (RCC) cell proliferation, migration, and invasion via inhibition of cadherin 2 (CDH2). A: Cell viability in response to overexpression (oe)-CDH2 alone or miR-411 mimic and oe-CDH2 treatment assessed by the MTT assay. B and C: The ability of cell colony formation in response to oe-CDH2 alone or miR-411 mimic and oe-CDH2 treatment assessed by clonogenic assay. D and E: Migration of cells transfected with oe-CDH2 alone or miR-411 mimic and oe-CDH2 evaluated by scratch test (the white dotted line in the figure indicates location of cell migration at the current time). $\mathbf{F}$ and $\mathbf{G}$ : Cell invasion in response to oe-CDH2 alone or miR-411 mimic and oe-CDH2 treatment evaluated by Transwell assay. Comparisons between two groups were performed using the unpaired $t$-test or correlation analysis. Comparisons among multiple groups were assessed by oneway analysis of variance or repeated-measures analysis of variance. The experiment was repeated three times independently. data are expressed as means \pm SD. ${ }^{*} P<0.05$ versus negative control (NC) mimic plus oe-NC transfected cells; ${ }^{\dagger} P<0.05$ versus miR-411 mimic plus oe-NC transfected cells. Original magnification: $\times 100(\mathbf{D}) ; \times 200(\mathbf{F})$. Scale bar $=50 \mu \mathrm{m}(\mathbf{F})$. si-, small interfering.

CDH2 in the presence of si-circ_000926. Tumor volume and weight in nude mice decreased markedly by inoculation of A498 cells transfected with si-circ_000926. The effects were reversed when si-circ_000926 was inhibited by miR411 or with elevation of $\mathrm{CDH} 2$ (Figure 12, A and B). These results suggest that restoration of $\mathrm{CDH} 2$ or reduction of miR-411 eliminates the si-circ_000926-caused inhibitory effects on tumor growth of A498 cells.

In addition, the positive rates of E-cadherin and vimentin proteins in the tumors of nude mice were determined by immunohistochemistry. Results indicate that the positive rates of vimentin proteins are decreased, whereas those of E-cadherin are increased in mice inoculated with A498 cells stably transfected with si-circ_000926. In contrast, the reductions in vimentin and elevation in Ecadherin were all eliminated by inhibition of miR-411 or elevation of $\mathrm{CDH} 2$ (Figure 12C). These results suggest that down-regulating circ_000926 exerted inhibitory effects on EMT and metastasis of RCC through regulating miR-411 and $\mathrm{CDH} 2$.

\section{Discussion}

In this study, a series of functional assays were conducted in RCC cells and nude mouse RCC models established to explore the biological implications of the circ_000926/miR$411 / \mathrm{CDH} 2$ axis in RCC. The results indicated that circ_000926 may function as a miR-411 sponge to upregulate $\mathrm{CDH} 2$ expression, thereby affecting the progression of RCC.

RT-qPCR revealed an augmented expression of circ_000926 in RCC tissues and cells. circRNA is a type of noncoding RNA, whereby its aberrant and unregulated expression has been associated with various diseases, such as atherosclerosis, nervous system disorders, and cancers. ${ }^{24}$ 

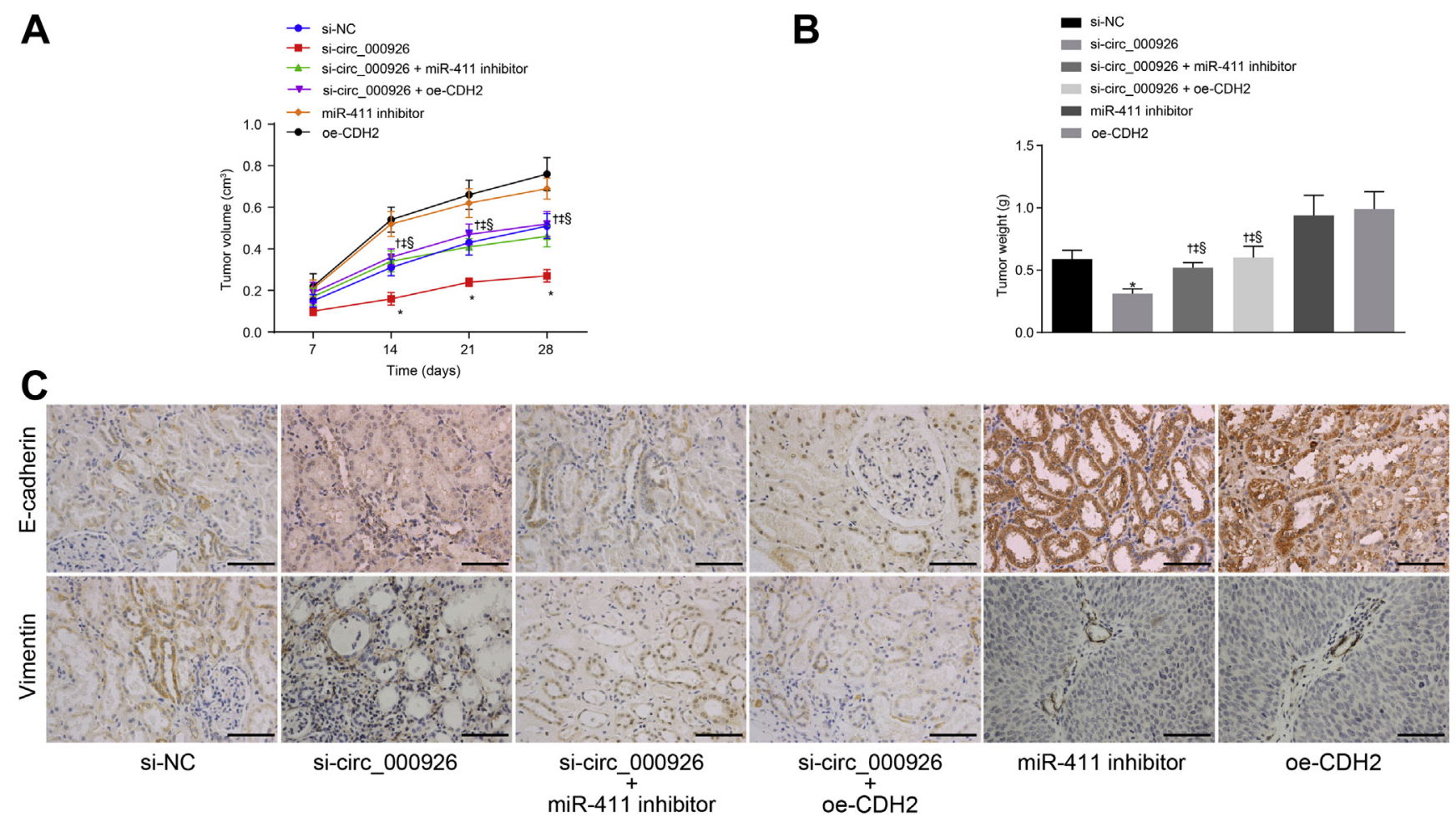

miR-411 inhibitor

oe- $\mathrm{CDH} 2$

Figure 12 Circular RNA 000926 (circ_000926) inhibition hinders tumor growth in nude mice through regulation of miRNA-411 (miR-411) and cadherin 2 (CDH2). A and B: Xenograft tumors and quantitative analysis of tumor mass after injection of A498 cells transfected with miR-411 inhibitor or overexpression (oe)-CDH2 in the presence of small interfering circ_000926 (si-circ_000926). C: Positive expression rate of E-cadherin and vimentin proteins in mice injected with A498 cells stably transfected with miR-411 inhibitor or oe-CDH2 in the presence of si-circ_000926 determined by immunohistochemistry. Comparisons among multiple groups were assessed by one-way analysis of variance or repeated-measures analysis of variance. Data are expressed as means \pm SD. $n=6$. ${ }^{*} P<0.05$ versus mice injected with si-negative control (NC) transfected cells; ${ }^{\dagger} P<0.05$ versus mice injected with si-circ_000926 transfected cells; ${ }^{\ddagger} P<0.05$ versus mice injected with miR-411 inhibitor transfected cells; ${ }^{\S} P<0.05$ versus mice injected with oe-CDH2-transfected cells. Original magnification, $\times 400$ (C). Scale bar $=50 \mu \mathrm{m}(\mathbf{C})$.

Recently, several circRNAs are documented to be implicated in the tumorigenesis of RCC. For example, hsa_circ_0001451 is observed to be highly expressed in clear cell RCC tissues, and its knockdown can accelerate tumor growth in vitro. ${ }^{25}$ Another circRNA known as circPCNXL2 is significantly up-regulated and associated with poor survival in patients with clear cell RCC. ${ }^{26}$ However, the full understanding of circRNAs function in RCC progression is limited. To further evaluate the molecular mechanisms of circRNAs and their roles in cellular functions and nude mice tumor formation, circ_000926 was silenced, and a decrease in RCC cell proliferation, colony formation, cell migration, cell invasion, and tumorigenesis was observed, accompanied with induced cell apoptosis.

This study also found the antitumor functions of miR-411 in the development of RCC. Consistent with our results, miR-411 is proposed as a tumor suppressor in RCC whereby the RT-qPCR assay reveals its down-regulation in RCC cell lines and tissues. ${ }^{12}$ In addition, miR-411 functions as an anti-tumor miRNA in breast cancer and non-smallcell lung cancer. ${ }^{27,28}$ In this study, RNA pull down, RNA capture, and RT-qPCR assays confirmed an interaction between circ_00926 and miR-411. circRNAs function as efficient miRNA sponges that can also bind to RNA- associated proteins to exert their regulatory effects on gene expression. ${ }^{29} \mathrm{~A}$ recent investigation revealed that another circRNA, circ_001569, acts as a sponge of miR-411 to facilitate proliferation and invasion of hepatocellular carcinoma cells. ${ }^{30}$ This study also suggested that miR-411 inhibition was able to reverse the anti-tumor effects of circ_000926 silencing in RCC, which supports the fact that circ_000926 sponges miR-411 to regulate RCC progression.

In this study, it was further observed how miR-411 could down-regulate $\mathrm{CDH} 2$. Functional investigations confirmed that $\mathrm{CDH} 2$ overexpression can eliminate the inhibitory effects of miR-411 on RCC progression in vitro. As a major maker of EMT, $\mathrm{CDH} 2$ appears to be associated with the malignant potential and aggressiveness of RCC. ${ }^{16,31}$ EMT can increase tumor cell activity and play a key role in cell invasion and metastasis in a cascade of cancers, including RCC. $^{32}$ In the present study, functional investigations confirmed that restoration of miR-411 function can eliminate the in vitro promoting effects of $\mathrm{CDH} 2$ overexpression on $\mathrm{RCC}$ progression. In addition, $\mathrm{CDH} 2$ also functions as downstream target of a few miRNAs. ${ }^{33}$ Specifically, miR194 is capable of suppressing growth and migration of osteosarcoma cells through negatively targeting $\mathrm{CDH} 2 .^{34}$ miR-124 can remarkably diminish the expression of 
$\mathrm{CDH} 2$ and inhibit the proliferation and invasion together with EMT in non-small-cell lung cancer cells. ${ }^{35}$ Through gain-and-loss functional experiments, circ_000926 was found to act as a ceRNA for miR-411 to increase CDH2 levels in RCC, whereby circ_000926 mediates the developmental process of RCC. This study provided evidence that restoration of $\mathrm{CDH} 2$ or reduction of miR-411 was able to eliminate the circ_000926 silencing-caused inhibition on RCC progression. Similarly, circ_000284 stimulates proliferative and invasive abilities of cervical cancer cells, which yet can be abolished by miR-506 enhancement or Snail-2 silencing. ${ }^{36}$ However, whether other known miRNAs that target CDH2 could be regulated by circ_000926 is warranted for further investigation.

Overall, this study provides evidence of how circ_000926 silencing may prevent the development and progression of RCC. We propose a mechanism by which circ_000926 acts as an miRNA sponge to directly inhibit miR-411 and subsequently up-regulate $\mathrm{CDH} 2$. Therefore, silencing of circ_000926 could be a promising direction to look toward and develop a novel treatment strategy for patients with RCC. However, because of the lack of data and reports on the clinical practical applications of circRNA-targeted therapy in RCC, further investigation is still required to explore the strategies in targeting circ_000926 in RCC.

\section{Acknowledgments}

We thank the reviewers for helpful comments.

\section{References}

1. Ljungberg B, Campbell SC, Choi HY, Jacqmin D, Lee JE, Weikert S, Kiemeney LA: The epidemiology of renal cell carcinoma. Eur Urol 2011, 60:615-621

2. Bhatt JR, Finelli A: Landmarks in the diagnosis and treatment of renal cell carcinoma. Nat Rev Urol 2014, 11:517-525

3. Ricketts CJ, Crooks DR, Sourbier C, Schmidt LS, Srinivasan R, Linehan WM: SnapShot: renal cell carcinoma. Cancer Cell 2016, 29: 610-610.e1

4. Ljungberg B, Cowan NC, Hanbury DC, Hora M, Kuczyk MA, Merseburger AS, Patard JJ, Mulders PF, Sinescu IC; European Association of Urology Guideline G: EAU guidelines on renal cell carcinoma: the 2010 update. Eur Urol 2010, 58:398-406

5. Fisher R, Gore M, Larkin J: Current and future systemic treatments for renal cell carcinoma. Semin Cancer Biol 2013, 23:38-45

6. Barata PC, Rini BI: Treatment of renal cell carcinoma: current status and future directions. CA Cancer J Clin 2017, 67:507-524

7. Maher ER: Genomics and epigenomics of renal cell carcinoma. Semin Cancer Biol 2013, 23:10-17

8. Chen LL: The biogenesis and emerging roles of circular RNAs. Nat Rev Mol Cell Biol 2016, 17:205-211

9. Hansen TB, Jensen TI, Clausen BH, Bramsen JB, Finsen B, Damgaard CK, Kjems J: Natural RNA circles function as efficient microRNA sponges. Nature 2013, 495:384-388

10. Wang Y, Mo Y, Gong Z, Yang X, Yang M, Zhang S, Xiong F, Xiang B, Zhou M, Liao Q, Zhang W, Li X, Li X, Li Y, Li G, Zeng Z, Xiong W: Circular RNAs in human cancer. Mol Cancer 2017, 16:25
11. Rupaimoole R, Calin GA, Lopez-Berestein G, Sood AK: miRNA deregulation in cancer cells and the tumor microenvironment. Cancer Discov 2016, 6:235-246

12. Zhang X, Zhang M, Cheng J, Lv Z, Wang F, Cai Z: MiR-411 functions as a tumor suppressor in renal cell cancer. Int J Biol Markers 2017, 32:e454-e460

13. Guo L, Yuan J, Xie N, Wu H, Chen W, Song S, Wang X: miRNA411 acts as a potential tumor suppressor miRNA via the downregulation of specificity protein 1 in breast cancer. Mol Med Rep 2016, 14:2975-2982

14. Zhao J, Xu J, Zhang R: MicroRNA-411 inhibits malignant biological behaviours of colorectal cancer cells by directly targeting PIK3R3. Oncol Rep 2018, 39:633-642

15. Terada N, Karim MR, Izawa T, Kuwamura M, Yamate J: Immunolocalization of beta-catenin, E-cadherin and $\mathrm{N}$-cadherin in neonate and adult rat kidney. J Vet Med Sci 2017, 79:1785-1790

16. Alimperti S, Andreadis ST: $\mathrm{CDH} 2$ and $\mathrm{CDH} 11$ act as regulators of stem cell fate decisions. Stem Cell Res 2015, 14:270-282

17. Behnes CL, Hemmerlein B, Strauss A, Radzun HJ, Bremmer F: $\mathrm{N}$-cadherin is differentially expressed in histological subtypes of papillary renal cell carcinoma. Diagn Pathol 2012, 7:95

18. Gautier L, Cope L, Bolstad BM, Irizarry RA: affy-analysis of Affymetrix GeneChip data at the probe level. Bioinformatics 2004, 20:307-315

19. Smyth GK: Linear models and empirical Bayes methods for assessing differential expression in microarray experiments. Stat Appl Genet Mol Biol 2004, 3. Article3

20. Chen P, Zhao L, Pan X, Jin L, Lin C, Xu W, Xu J, Guan X, Wu X, Wang Y, Yang S, Wang T, Lai Y: Tumor suppressor microRNA-136$5 p$ regulates the cellular function of renal cell carcinoma. Oncol Lett 2018, 15:5995-6002

21. Kim CJ, Terado $T$, Tambe Y, Mukaisho KI, Sugihara H, Kawauchi A, Inoue $\mathrm{H}$ : Anti-oncogenic activities of cyclin D1b siRNA on human bladder cancer cells via induction of apoptosis and suppression of cancer cell stemness and invasiveness. Int $\mathrm{J}$ Oncol 2018, 52:231-240

22. Dudekula DB, Panda AC, Grammatikakis I, De S, Abdelmohsen K, Gorospe M, CircInteractome: A web tool for exploring circular RNAs and their interacting proteins and microRNAs. RNA Biol 2016, 13: $34-42$

23. Garcia DM, Baek D, Shin C, Bell GW, Grimson A, Bartel DP: Weak seed-pairing stability and high target-site abundance decrease the proficiency of 1sy-6 and other microRNAs. Nat Struct Mol Biol 2011, 18:1139-1146

24. Xu T, Wu J, Han P, Zhao Z, Song X: Circular RNA expression profiles and features in human tissues: a study using RNA-seq data. BMC Genomics 2017, 18:680

25. Wang G, Xue W, Jian W, Liu P, Wang Z, Wang C, Li H, Yu Y, Zhang D, Zhang C: The effect of Hsa_circ_0001451 in clear cell renal cell carcinoma cells and its relationship with clinicopathological features. J Cancer 2018, 9:3269-3277

26. Zhou B, Zheng P, Li Z, Li H, Wang X, Shi Z, Han Q: CircPCNXL2 sponges miR-153 to promote the proliferation and invasion of renal cancer cells through upregulating ZEB2. Cell Cycle 2018, 17: 2644-2654

27. Zhang Y, Xu G, Liu G, Ye Y, Zhang C, Fan C, Wang H, Cai H, Xiao R, Huang Z, Luo Q: miR-411-5p inhibits proliferation and metastasis of breast cancer cell via targeting GRB2. Biochem Biophys Res Commun 2016, 476:607-613

28. Xia LH, Yan QH, Sun QD, Gao YP: MiR-411-5p acts as a tumor suppressor in non-small cell lung cancer through targeting PUM1. Eur Rev Med Pharmacol Sci 2018, 22:5546-5553

29. Han B, Chao J, Yao H: Circular RNA and its mechanisms in disease: from the bench to the clinic. Pharmacol Ther 2018, 187:31-44

30. Liu H, Xue L, Song C, Liu F, Jiang T, Yang X: Overexpression of circular RNA circ_001569 indicates poor prognosis in hepatocellular carcinoma and promotes cell growth and metastasis by sponging 
miR-411-5p and miR-432-5p. Biochem Biophys Res Commun 2018, 503:2659-2665

31. Shimazui T, Kojima T, Onozawa M, Suzuki M, Asano T, Akaza H: Expression profile of $\mathrm{N}$-cadherin differs from other classical cadherins as a prognostic marker in renal cell carcinoma. Oncol Rep 2006, 15:1181-1184

32. Chen D, Gassenmaier M, Maruschke M, Riesenberg R, Pohla H, Stief CG, Zimmermann W, Buchner A: Expression and prognostic significance of a comprehensive epithelial-mesenchymal transition gene set in renal cell carcinoma. J Urol 2014, 191:479-486

33. Liu X, Duan H, Zhang HH, Gan L, Xu Q: Integrated data set of microRNAs and mRNAs involved in severe intrauterine adhesion. Reprod Sci 2016, 23:1340-1347
34. Miao J, Wang W, Wu S, Zang X, Li Y, Wang J, Zhan R, Gao M, Hu M, Li J, Chen S: miR-194 suppresses proliferation and migration and promotes apoptosis of osteosarcoma cells by targeting $\mathrm{CDH} 2$. Cell Physiol Biochem 2018, 45:1966-1974

35. Ma T, Zhao Y, Wei K, Yao G, Pan C, Liu B, Xia Y, He Z, Qi X, Li Z, Wang J, Shao Y: MicroRNA-124 functions as a tumor suppressor by regulating $\mathrm{CDH} 2$ and epithelial-mesenchymal transition in non-small cell lung cancer. Cell Physiol Biochem 2016, 38:1563-1574

36. Ma HB, Yao YN, Yu JJ, Chen XX, Li HF: Extensive profiling of circular RNAs and the potential regulatory role of circRNA-000284 in cell proliferation and invasion of cervical cancer via sponging miR-506. Am J Transl Res 2018, 10:592-604 